\title{
A study of asteroid pole-latitude distribution based on an extended set of shape models derived by the lightcurve inversion method ${ }^{\star}$
}

\author{
J. Hanuš ${ }^{1}$, J. Ďurech ${ }^{1}$, M. Brož ${ }^{1}$, B. D. Warner ${ }^{2}$, F. Pilcher ${ }^{3}$, R. Stephens ${ }^{4}$, J. Oey ${ }^{5}$, L. Bernasconi ${ }^{6}$, S. Casulli ${ }^{7}$, \\ R. Behrend ${ }^{8}$, D. Polishook ${ }^{9}$, T. Henych ${ }^{10}$, M. Lehký ${ }^{11}$, F. Yoshida ${ }^{12}$, and T. Ito ${ }^{12}$ \\ 1 Astronomical Institute, Faculty of Mathematics and Physics, Charles University in Prague, V Holešovičkách 2, 18000 Prague, \\ Czech Republic \\ e-mail: hanus.home@gmail.com \\ 2 Palmer Divide Observatory, 17995 Bakers Farm Rd., Colorado Springs, CO 80908, USA \\ 34438 Organ Mesa Loop, Las Cruces, NM 88011, USA \\ ${ }^{4}$ Goat Mountain Astronomical Research Station, 11355 Mount Johnson Court, Rancho Cucamonga, CA 91737, USA \\ 5 Kingsgrove, NSW, Australia \\ ${ }^{6}$ Observatoire des Engarouines, 84570 Mallemort-du-Comtat, France \\ 7 Via M. Rosa 1, 00012 Colleverde di Guidonia, Rome, Italy \\ 8 Geneva Observatory, 1290 Sauverny, Switzerland \\ 9 Benoziyo Center for Astrophysics, The Weizmann Institute of Science, Rehovot 76100, Israel \\ 10 Astronomical Institute, Academy of Sciences of the Czech Republic, Fričova 1, 25165 Ondřejov, Czech Republic \\ 11 Severni 765, 50003 Hradec Kralove, Czech republic \\ 12 National Astronomical Observatory, Osawa 2-21-1, Mitaka, 181-8588 Tokyo, Japan
}

Received 17 February 2011 / Accepted 13 April 2011

\begin{abstract}
Context. In the past decade, more than one hundred asteroid models were derived using the lightcurve inversion method. Measured by the number of derived models, lightcurve inversion has become the leading method for asteroid shape determination.

Aims. Tens of thousands of sparse-in-time lightcurves from astrometric projects are publicly available. We investigate these data and use them in the lightcurve inversion method to derive new asteroid models. By having a greater number of models with known physical properties, we can gain a better insight into the nature of individual objects and into the whole asteroid population.

Methods. We use sparse photometry from selected observatories from the AstDyS database (Asteroids - Dynamic Site), either alone or in combination with dense lightcurves, to determine new asteroid models by the lightcurve inversion method. We investigate various correlations between several asteroid parameters and characteristics such as the rotational state and diameter or family membership. We focus on the distribution of ecliptic latitudes of pole directions. We create a synthetic uniform distribution of latitudes, compute the method bias, and compare the results with the distribution of known models. We also construct a model for the long-term evolution of spins.

Results. We present 80 new asteroid models derived from combined data sets where sparse photometry is taken from the AstDyS database and dense lightcurves are from the Uppsala Asteroid Photometric Catalogue (UAPC) and from several individual observers. For 18 asteroids, we present updated shape solutions based on new photometric data. For another 30 asteroids we present their partial models, i.e., an accurate period value and an estimate of the ecliptic latitude of the pole. The addition of new models increases the total number of models derived by the lightcurve inversion method to $\sim 200$. We also present a simple statistical analysis of physical properties of asteroids where we look for possible correlations between various physical parameters with an emphasis on the spin vector. We present the observed and de-biased distributions of ecliptic latitudes with respect to different size ranges of asteroids as well as a simple theoretical model of the latitude distribution and then compare its predictions with the observed distributions. From this analysis we find that the latitude distribution of small asteroids $(D<30 \mathrm{~km})$ is clustered towards ecliptic poles and can be explained by the YORP thermal effect while the latitude distribution of larger asteroids $(D>60 \mathrm{~km})$ exhibits an evident excess of prograde rotators, probably of primordial origin.
\end{abstract}

Key words. minor planets, asteroids: general - thechniques: photometric - methods: numerical - methods: statistical

\section{Introduction}

The lightcurve inversion method (LI) is a powerful tool that allows us to derive basic physical properties of asteroids (the rotational state and the shape) from their disk-integrated photometry (see Kaasalainen \& Torppa 2001; Kaasalainen et al. 2001, 2002). This photometry can be dense-in-time, which typically consists of tens to a few hundreds of individual data points

* Tables 3-6 are available in electronic form at http: //www . aanda.org observed during one revolution. This is in contrast to sparse-intime, where the typical separation of individual measurements is large compared to the rotation period. For sparse data, we usually have a few measurements per night, such as in the case of astrometric sky surveys. In the following text, we use the terms "dense lightcurves" and "sparse lightcurves".

To obtain a unique spin and shape solution, we need a set of at least a few tens of dense lightcurves observed during at least three apparitions. Based on simulated photometric data and the survey cadence of the Panoramic Survey Telescope And Rapid 
Response System (Pan-STARRS), Kaasalainen (2004) showed that we can also use only sparse data for the inversion technique. In this case, a unique model can be derived from more than about one hundred calibrated measurements observed during 3-5 years if the photometric accuracy is better than $\sim 5 \%$ (Durech et al. 2005, 2007). Sparse data available so far are not that accurate. Nevertheless, for many asteroids with high lightcurve amplitudes, it is possible to derive their models from current sparse data (see Sect. 2 for more details). We can also combine sparse and dense data to derive models. First results from this approach were presented by Durech et al. (2009), where sparse data from the US Naval Observatory in Flagstaff (USNO) were used.

Currently (January 2011), there are 113 models of asteroids derived by the lightcurve inversion method; most of them are stored in the Database of Asteroid Models from Inversion Techniques (DAMIT ${ }^{1}$, Durech et al. 2010). Most of these models were derived from dense lightcurves. Only 24 of them were computed from combined dense and sparse data (Ďurech et al. 2009). The AstDyS database (Asteroids - Dynamic Site ${ }^{2}$ ), which contains data from astrometric projects, is another possible source of sparse data. However, most of the data are not accurate enough to be used for inversion alone. On the other hand, even noisy sparse data in combination with a few dense lightcurves can give us, in many cases, a unique solution (Durech et al. 2007). The aim of our work was to gather these data, keep only those that were useful, and then combine them with dense lightcurves in the lightcurve inversion method.

Dense data are best used to define the rotational period and constrain the period interval that must be searched during the model computation (see Sect. 3.1 for more details). On the other hand, sparse data usually cover a long time interval, typically over several apparitions, and carry information about brightness variations for different geometries, which constrains the pole directions.

A priori information about rotational periods of asteroids plays an important role in the process of model determination. When an approximate period is known, we search for the solution near this value (details in Sect. 3.1) and thus save considerable computational time. We use the latest update of the Minor Planet Lightcurve Database ${ }^{3}$ published by Warner et al. (2009) to check for previously derived periods. For many asteroids, there are only a few sparse lightcurves from different astrometric observatories available but no dense lightcurves. In these cases, we must scan the whole interval of expected period values $(2-30 \mathrm{~h})$. This approach is time-consuming and there is no guarantee that the correct period will lie in the scanned interval.

The knowledge of rotational states of asteroids is fundamental for understanding the history of the Solar System, specifically the accretion of planets or the collisional processes. For example, it was presumed that due to collisional evolution, the spin-vector distribution of main belt asteroids (MBAs) should be nearly isotropic, possibly with a small excess of prograde spins (Davis et al. 1989). Johansen \& Lacerda (2010) performed a hydrodynamical simulation of the accretion of pebbles and rocks onto protoplanets and speculated that the trend of prograde rotators among the largest asteroids is primordial.

\footnotetext{
${ }^{1}$ http://astro.troja.mff.cuni.cz/projects/asteroids3D

2 http://hamilton.dm.unipi.it/

${ }^{3}$ http://cfa-www . harvard.edu/iau/lists/LightcurveDat.html
}

First statistical analyses of the spin-vector distribution were presented by Magnusson $(1986,1990)$ and Drummond et al. (1988, 1991), later by Pravec et al. (2002) and Skoglöv \& Erikson (2002). They all observed a lack of poles close to the ecliptic plane. Kryszczyńska et al. (2007) used more objects in the analysis, finding that the distribution was strongly anisotropic with a moderate excess of prograde spins in the limited size range from 100 to $150 \mathrm{~km}$. Interpretation of this depopulation of poles close to the ecliptic plane is still unclear. Probable candidates are selection effects, the role of inclination, the YORP effect ${ }^{4}$ (Rubincam 2000; Vokrouhlický et al. 2003), or a combination of these. The YORP effect acts only on small bodies with $D \lesssim 40 \mathrm{~km}$. Asteroids with these sizes have nonMaxwellian spin rate distribution (Pravec \& Harris 2000) and is particularly evident for asteroids with $D<14 \mathrm{~km}$ (Warner et al. 2009). It is believed that the YORP effect is responsible for this trend since it can either spin up or spin down an irregularlyshaped asteroid on the timescale shorter than the typical time between collisions and also affects the obliquity of spin axes (Rubincam 2000; Bottke et al. 2006).

In the near Earth asteroids (NEAs) population, the latitude distribution of poles is different from that of MBAs (La Spina et al. 2004; Kryszczyńska et al. 2007), i.e., there is a significant excess of retrograde spins probably caused by the transport mechanism of MBAs to Earth-crossing space by gravitational resonances and the Yarkovsky effect ${ }^{5}$ (Morbidelli \& Vokrouhlický 2003). There is no statistically significant clustering in the longitude of poles of either MBAs or NEAs (Kryszczyńska et al. 2007).

As the number of asteroid models with known physical properties grows, we can have a better insight into the nature of individual objects and into the asteroid population as a whole. In Sect. 2, we describe available dense and sparse photometric data and the selection of astrometric observatories with quality sparse data. In Sect. 3, we present new asteroid models derived from combined photometric data sets or from sparse data alone, mentioning a few individual objects and define several procedures on how to test the reliability of new models. In Sect. 4, we present a statistical analysis of asteroid physical parameters that we derived using the lightcurve inversion method or adopted from different sources (proper elements from the AstDyS database, diameters from IRAS, ...). We also present results of a numerical simulation that allowed us to estimate the bias in pole directions of the lightcurve inversion method. Using these results, we then corrected the observed pole distributions for this effect. Finally, in order to explain the observed latitude distributions, we present a simple theoretical model of the latitude distribution in Sect. 5.

\section{Photometric data}

The main source of dense photometric lightcurves is the Uppsala Asteroid Photometric Catalogue (UAPC, Lagerkvist et al. 1987; Piironen et al. 2001), where the lightcurves for about 1000 asteroids are stored. We also used data from several individual observers (Table 6).

Sparse photometry was first used in combination with dense data for lightcurve inversion by Durech et al. (2009). These sparse data were from the USNO-Flagstaff station and had a typical photometric uncertainty of $\sim 8-10 \%$. Other sparse photometric measurements are produced by many astrometric surveys,

\footnotetext{
4 Yarkovsky-O'Keefe-Radzievskii-Paddack effect, a torque caused by the recoil force from anisotropic thermal emission.

5 A thermal force acting on a rotating asteroid.
} 
Table 1. Comparison of estimated characteristics of residuals for 13 selected observatories: mode, " $F W H M$ " and median.

\begin{tabular}{rrcccccc}
\hline \hline Obs & $N_{\mathrm{LC}}$ & Mode & $F W H M$ & Median & $\overline{N_{\mathrm{P}}}$ & Weight & Observatory name \\
\hline 608 & 2459 & 0.26 & 0.20 & 0.27 & 37 & 0 & Haleakala-AMOS \\
644 & 2567 & 0.22 & 0.22 & 0.24 & 36 & 0 & Palomar Mountain/NEAT \\
683 & 218 & 0.18 & 0.25 & 0.20 & 39 & 0 & Goodricke-Pigott Observatory, Tucson \\
689 & 1970 & 0.14 & 0.12 & 0.15 & 118 & 0.3 & U.S. Naval Observatory, Flagstaff \\
691 & 1893 & 0.23 & 0.22 & 0.24 & 39 & 0 & Steward Observatory, Kitt Peak-Spacewatch \\
699 & 546 & 0.17 & 0.11 & 0.18 & 33 & 0.1 & Lowell Observatory-LONEOS \\
703 & 8350 & 0.17 & 0.16 & 0.19 & 54 & 0.15 & Catalina Sky Survey \\
704 & 8333 & 0.42 & 0.17 & 0.42 & 311 & 0 & Lincoln Laboratory ETS, New Mexico \\
950 & 80 & 0.14 & 0.11 & 0.15 & 180 & 0.15 & La Palma \\
E12 & 1354 & 0.14 & 0.16 & 0.15 & 41 & 0.1 & Siding Spring Survey \\
G96 & 1810 & 0.14 & 0.22 & 0.17 & 43 & 0.1 & Mt. Lemmon Survey \\
H07 & 161 & 0.20 & 0.18 & 0.23 & 47 & 0 & 7300 Observatory, Cloudcroft \\
Hip & 49 & 0.10 & 0.10 & 0.11 & 53 & 0.3 & Hipparcos satellite \\
\hline
\end{tabular}

Notes. For each observatory, the table gives also the number of sparse lightcurves $N_{\mathrm{LC}}$, and the average number of data points for a single lightcurve $\overline{N_{\mathrm{P}}}$.

but mostly as a by-product. In most cases, asteroid magnitudes are given to only one decimal place, i.e., the accuracy is $0.1 \mathrm{mag}$ at best. Whether or not this is sufficient for a unique shape determination for reasonable number of asteroids can be deduced from asteroids lightcurve amplitude distribution. We used lightcurve amplitude data for 2500 asteroids from the Minor Planet Lightcurve Database (Warner et al. 2009) and found that the mean lightcurve amplitude is $\sim 0.3 \mathrm{mag}$. For $19 \%$ of asteroids, the amplitude is $\geq 0.5 \mathrm{mag}$. This means that, in principle, photometry with an accuracy of $\sim 0.1$ mag carries sufficient information about rotational states and shapes for a significant number of asteroids.

Our goal was to find out which observatories produce photometry suitable for lightcurve inversion and to use these data for determining new asteroid models. Through to September 2009 (the time of the data download), data for more than 350000 objects from almost 1500 observatories were archived on the AstDyS server. Some of the observatories contributed with only a few data points, while others contributed tens of thousands of photometric measurements (e.g., large sky surveys such as the Catalina Sky Survey, LONEOS, or Siding Spring Survey).

\subsection{Data reduction}

The quality of the sparse photometry archived on the AstDyS varies significantly. We investigated the photometry carefully by establishing criteria for its quality. Then, using those criteria, we choose only those data that were useful for inversion.

For each observatory, we extracted photometric data for the first 10000 numbered asteroids if there were at least 30 data points for a single lightcurve. We then transformed this photometry to the standard format used in lightcurve inversion: we computed geometry of observation (astrocentric ecliptic coordinates of the Sun and the Earth), corrected for light-time, normalized the brightness to the distance of $1 \mathrm{AU}$ from the Sun and the Earth, and excluded clear outliers.

For further investigation, we selected 13 observatories that fulfilled the condition of having data for more than $\sim 50$ asteroids. This resulted in almost 30000 sparse lightcurves for $\sim 9000$ asteroids. In the next step, we estimated mean uncertainties of individual observatories and, based on these uncertainties, we assigned a relative weight to the data from each observatory. In this process, we assumed that the brightness vs. solar phase angle relation can be fitted with a simple relation for each sparse lightcurve:

$f(\alpha)=\cos ^{2}\left(\frac{\alpha}{2}\right)\left[a \exp \left(-\frac{\alpha}{b}\right)+c \alpha+d\right]$,

where $\alpha$ is the solar phase angle ${ }^{6}$ and $a, b, c$ and $d$ are free parameters. Then, we constructed a histogram of residuals (rms) for each observatory comparing actual data against the model given by Eq. (1). Four examples are plotted in Fig. 1. The dispersion is caused by observational uncertainties and by the amplitudes of the lightcurves. From these histograms, we estimated the " $F W H M$ "7 values and the most frequent residual (the mode); median values of the residual distributions for each location (Table 1) were computed. Observatories with a high median or " $F W H M$ " value ( $\gtrsim 0.2 \mathrm{mag}$ for both) are not suitable for the lightcurve inversion (e.g. observatory 691 in Fig. 1). Data from only seven observatories, listed in Table 1 with non-zero weights, had sufficient accuracy and so could be used for modeling. Based on the values of medians and " $F W H M$ s", we estimated a weight for the photometric data from each observatory relative to dense data, which has a unity weight (see Table 1). We assumed that the typical accuracy of dense lightcurves is $\sim 0.02$ mag.

The USNO in Flagstaff (MPC code 689) and the Hipparcos satellite are clearly the best observatories with respect to photometric accuracy. Other observatories are less accurate but they still hold some information about rotational states and shapes of asteroids. For any given asteroid, we have typically 2-4 sparse lightcurves from different observatories covering the last 10-15 years. Data from USNO were already used by Durech et al. (2009). We updated those data along with adding another 20-30\% of new data from the years 2008 and 2009 if there was an apparition for the asteroid.

\section{Results}

\subsection{Models of asteroids}

Our lightcurve inversion (LI) method is based on the optimization of unknown parameters of the shape (modeled as a convex hull), the rotational state, and the scattering law (see Kaasalainen \& Torppa 2001; Kaasalainen et al. 2001). The parameter space have many local minims. Since LI is a gradient-based method

\footnotetext{
6 The Sun-asteroid-Earth angle.

7 The width of the distribution in the half of its maximum.
} 

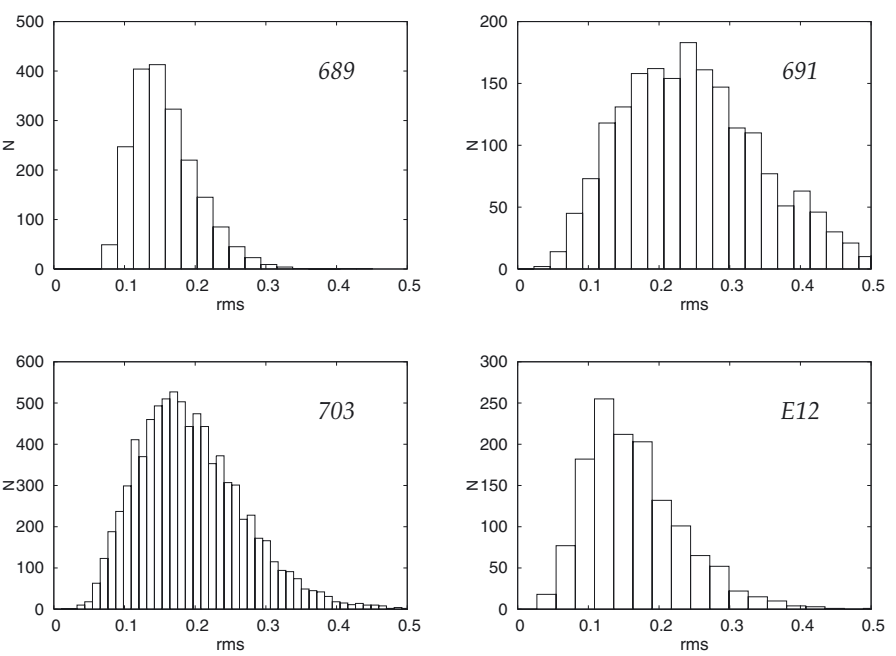

Fig. 1. Four histograms of residuals comparing actual data against the model given by Eq. (1) of all sparse lightcurves belonging to the following observatories: 689 USNO, 691 Steward Observatory, 703 Catalina Sky Survey and E12 Siding Spring Survey. Number of bins is $\sim \sqrt{N}$, where $N$ is the total number of sparse lightcurves used for histogram construction.

that converges to a local minimum near the initial choice of parameter values, it is critical to find the global minimum for the data set and then do the modeling. Finding a global minimum involves a systematic search through all relevant parameter values. Each model corresponding to a particular local minimum is characterized by a single value $\chi^{2}$, which corresponds to the quality of the fit.

A unique solution is defined as follows: (i) the best period has at least $10 \%$ lower $\chi^{2}$ than all other periods in the scanned interval; (ii) for this period, there is only one pole solution with at least $10 \%$ lower $\chi^{2}$ than the others (with a possible ambiguity for $\lambda \pm 180^{\circ}$ ); and (iii) this solution fulfills our additional tests (see Sect. 3.3).

The most time-consuming part of the lightcurve inversion method is scanning through all periods within a chosen interval, which we determined by using the periods and reliability codes given in the Minor Planet Lightcurve Database ${ }^{8}$. For each asteroid, we scanned an interval centered at the reported period value $P$ with a range of $\pm 1 \%, \pm 5 \%$ and $\pm 20 \%$ of $P$ for reliability codes 4,3 and 2, respectively. Half and double periods were tested later on.

We combined relative lightcurves from the UAPC and from individual observers together with sparse data obtained from the AstDyS site to create a data set for each asteroid. This gave us data sets for $\sim 2300$ asteroids (in $\sim 900$ cases there were only sparse data available) to which we then applied the lightcurve inversion method and then we ran the additional tests described in Sect. 3.3. We derived 80 new unique models, 16 of which are based only on sparse data. Basic characteristics of these models are listed in Table 3 . We estimated the uncertainty in the pole direction as $\pm 10-20^{\circ}$ based on previous results with limited data sets. As might be expected, the uncertainty seems dependent on the number of dense and sparse photometric data. The longitude uncertainty increases for higher latitudes because meridians on a $(\lambda, \beta)$-sphere are more dense with increasing latitude. These uncertainties are discussed in more detail in Sect. 4.3. The

\footnotetext{
8 http://cfa-www.harvard.edu/iau/lists/LightcurveDat.html, there is also an explanation and more details about the reliability codes too.
}

uncertainty of the rotational period depends on the time interval covered by observational data and is of the order of the last decimal place of period values $P$ in Tables $3-5$.

In some cases, we were able to determine a unique rotational period, but we had multiple pole solutions with similar ecliptic latitudes $\beta$. These models give us accurate period values and rough estimates of ecliptic latitudes $\beta$, which are also important parameters. In Table 4, we present results for 30 partial models, where $\beta$ is the mean value for all different models if the dispersion is smaller than $50^{\circ}$. We defined a parameter $\Delta=\left|\beta_{\max }-\beta_{\min }\right| / 2$ as being the estimated uncertainty of $\beta$, where $\beta_{\max }$ and $\beta_{\min }$ are the extremal values within all $\beta$.

All new unique shape models are now included in DAMIT.

\subsection{Comments to selected models}

In DAMIT there are several solutions designated as "preliminary". These models do not have a well-constrained pole solution or are based on combined data sets. For 18 of those asteroids we derived updated model solutions based on additional photometric data (see Table 5). The difference between the old and new model for asteroid (1223) Neckar was significant. The new model has a slightly different period, but the pole directions and shapes are nearly similar to the old model. The current data suggest a period of $P=7.82401 \mathrm{~h}$ (previous value was $P=7.82123 \mathrm{~h}$ ).

The asteroid (4483) Petofi was recently observed by Brian Warner. We derived a shape solution from three poor dense lightcurves and one sparse lightcurve from Catalina Sky Survey. Warner used these four lightcurves in combination with his new observations and also derived the shape model of Petofi (Warner 2011a). His period of $P=4.3330 \mathrm{~h}$ and pole direction $\left(90^{\circ}, 35^{\circ}\right)$ are close to our solution of $P=4.33299 \mathrm{~h}$ and $\left(107^{\circ}, 40^{\circ}\right)$.

The asteroid (832) Karin was also studied by Slivan \& Molnar (2010); their solution with $P=18.352 \mathrm{~h}$ and pole $\left(51^{\circ}\right.$ or $228^{\circ}, 41^{\circ}$ ) confirms our results, see Table 3 .

In past decades, occultations of stars by several asteroids were observed. These events give us additional information about the shape and can help resolve which mirror solutions of a model is the correct one. According to the recent work of Durech et al. (2011), asteroid occultation measurements prefer pole solutions of $\left(122^{\circ},-44^{\circ}\right)$ for $(10)$ Hygiea, $\left(347^{\circ}, 47^{\circ}\right)$ for (152) Atala, $\left(28^{\circ},-72^{\circ}\right)$ for (302) Clarissa, $\left(223^{\circ}, 67^{\circ}\right)$ for $(471)$ Papagena, and $\left(296^{\circ}, 41^{\circ}\right)$ for $(925)$ Alphonsina. Spin solutions preferred by asteroid occultations appear in bold font in Tables 3 and 5 .

\subsection{Models and method testing}

We constructed five additional tests to be sure that the new models are reliable. We performed the first two tests for all models. For models derived only from sparse data, which are presented for the first time, we performed three additional tests:

Inertia tensor of the shape model. The lightcurve inversion method we use assumes that asteroids are in a relaxed rotational state, which means that derived models should rotate around the axis with a maximum moment of inertia. For each derived shape, we computed principal moments using equations presented by Dobrovolskis (1996) and checked if the rotation axis was close to the principal axis of the maximum momentum of inertia. We rejected those models for which the angle between the spin axis and the axis with a maximum momentum of inertia was larger 
than $\pm 30^{\circ}$. However, this criterion is too strict for elongated models with similar sizes along the rotational axis and the axis that is both perpendicular to the rotational axis and is the minimal size of the model. In this case, the principal moments for these two axes are similar. Under these circumstances, the angle between the spin axis and the axis with a maximum momentum of inertia can be large even for realistic shapes and so we allowed these models to pass this test.

Half- and double-period models. In cases where we have only a few dense lightcurves for a given asteroid, it is easy to confuse the correct rotational period with its half or double value. When the a priori period value in the Minor Planet Lightcurve Database is uncertain, which corresponds to a low reliability code (see Sect. 3.1), it is reasonable to check if the half and double period value give a better fit. If the period was in doubt, we searched for a solution also around $2 P$ and $P / 2$; if the $\chi^{2}$ was lower than $1.1 \chi^{2}$ of the solution with period $P$, we rejected the model as unreliable.

Reduction of the number of sparse photometric data. In this test, we used only the sparse data sets for modeling. For 63 asteroids, this led to unique shape and spin state solutions (after performing the tests described above). For each solution, we randomly reduced the original amount of observed sparse data points to $90 \%$ and used these new limited data sets again in the lightcurve inversion. Our expectation was that we would not get a unique solution when using less data. This was true for five asteroids. These models, when using the full sparse data sets, are not necessarily wrong, but the amount of available data is probably just at the level when a unique solution can be derived. The important point of this test is that, for a given asteroid, we did not find two different but formally correct solutions when using the full versus reduced data sets. In Table 3, we present 16 models successfully derived only from sparse data that passed this test.

Models from sparse data vs. DAMIT. Here, we used previously derived models based only on relative photometry which are stored in DAMIT. As can seen in the previous test, sparse data are sometimes sufficient to produce a unique model of an asteroid. In 16 cases, we were able to derive a model for an asteroid which was already included in DAMIT and thus a model based on entirely different photometric data sets is available. These two independent models can be then compared and should be similar. We obtained similar resulting models for all 16 asteroids.

Models of "mock" objects. For each asteroid shape model derived only from sparse data, we created a set of ten "mock" objects of roughly the same appearance and spin state (see an example of such shape in Fig. 2). For these synthetic objects, we computed their photometric data using same epochs and geometries and with similar random noise level. These synthetic photometric data sets were then used in the lightcurve inversion method. A check that the original model using actual data is reliable is to be able to derive most of the models of the "mock" objects. The dispersions between the periods and pole directions of the "mock" objects represent the typical uncertainties of these parameters. For all studied asteroids, we were able to derive unique models for most of their "mock" objects. In all cases when we did not get a unique solution for the "mock" object, the best fit corresponded to the correct solution although other solutions could not be ruled out. The typical uncertainty in pole direction was $\pm 10^{\circ}$ and, for the period, $\sim 0.1$ times the difference between the two local minimums as determined by the period value and the time span of the data.

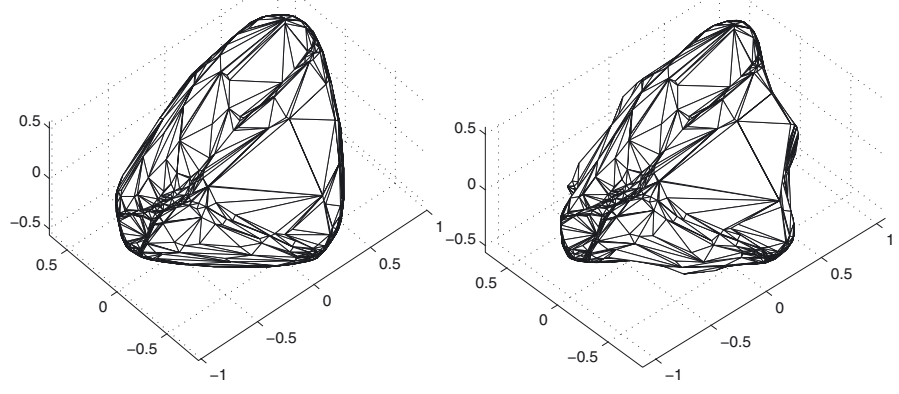

Fig. 2. Asteroid (810) Atossa: shape model (left panel) and an example of a "mock" shape model (right panel).

\section{An analysis of periods, poles and sizes of asteroid models}

Previous studies have been looking on spin state results using different techniques, e.g. amplitude-epoch, lightcurve inversion or radar methods. If there were multiple solutions for a given asteroid, the most probable one or simply the weighted mean was taken. However, this could cause systematic deviations. In our study, we used only results based on the lightcurve inversion method - i.e., unique and partial models presented in this work and models from the DAMIT. Our sample consists of 221 asteroid models: 80 new models, 18 updates for models from DAMIT, 30 new partial models, 84 models from DAMIT and 9 new models presented by Durech et al. (2011). Our sample consists of models for 206 MBAs, 10 NEAs, 3 Hungaria, 1 Trojan, and 1 Hilda and so a statistical study is only possible for the MBAs. In many cases there is an ambiguity in the pole direction since there are two, undistinguishable mirror solutions. For our statistical analysis we randomly chose only one.

In Fig. 3, we show (among other things to be discussed later) the relation between the proper semi-major axis and the proper eccentricity for asteroids in our sample and for all main belt asteroids. It is obvious that the positions of studied asteroids strongly correlate with the MBAs population and so derived models are not significantly biased with respect to orbits, e.g. they do not lie in the inner main belt. Several asteroids in Fig. 3 with semi-major axis $a>3.3$ AU belong to the Cybele group, e.g. (121) Hermione.

\subsection{Pole distribution analysis}

In the following study of spin axis directions, we did not use the Koronis family members because their spin states are correlated, i.e., their spin vectors are clustered towards two values of the obliquity (Slivan 2002). In Fig. 4a, we show the ecliptic latitude distribution of our MBA sample. As in all similar plots, the width of the latitude and longitude bins corresponds to equal surfaces on the $(\lambda, \beta)$-sphere (bins are equidistant in $\sin \beta$ for latitudes and in $\lambda$ for longitudes). We confirmed the expectation that there is a lack of asteroids with latitudes close to the ecliptic plane. The latitude distribution is clearly not symmetric: about half of the retrograde rotators have latitudes in the bin $\left(-53^{\circ},-90^{\circ}\right)$. On the other hand, less than a third of asteroids with prograde spins are in the corresponding bin $\left(53^{\circ}, 90^{\circ}\right)$. Moreover, the remaining prograde bins are more populated than the corresponding retrograde ones. From a detailed look at the plot we can see that there are up to $10 \%$ more prograde rotators among the MBAs.

In Fig. 5, we show the dependence of the ecliptic latitude $\beta$ of the pole direction on the diameter $D$ (most of the diameters 


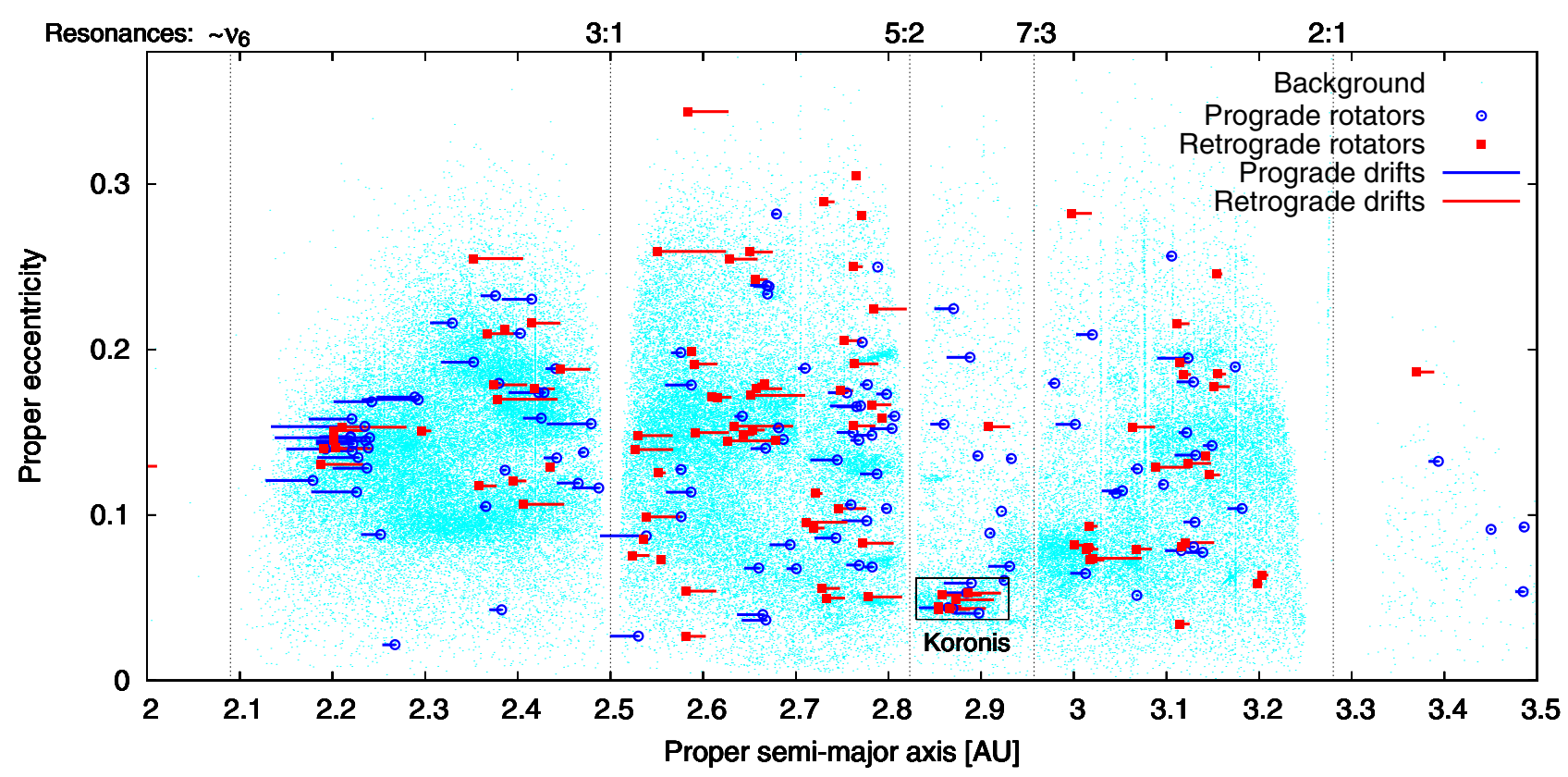

Fig. 3. Relation between the proper semi-major axis and the proper eccentricity for asteroids in our sample and for the first 100000 numbered asteroids for comparison. Main resonances are shown by dotted lines. Prograde rotators are plotted with blue circles and retrograde rotators with red squares. The horizontal lines represent for each asteroid its estimated past drift (i.e. where the asteroid came from) during the collisional lifetime computed with Eq. (7). Proper elements are from the AstDyS database.

used are based on the IRAS data (Tedesco et al. 2002) or occultations profiles and have an uncertainty $\pm 10 \%)$. Even for diameters $D \lesssim 50 \mathrm{~km}$, the clustering of the latitudes towards higher absolute values, and conversely, the depletion close to the ecliptic plane is obvious and markedly so for $D \lesssim 30 \mathrm{~km}$. Asteroids with larger diameters have a more isotropic distribution of latitudes and only a moderate excess of prograde. In Fig. 6, we plotted the latitude and longitude distributions of asteroids with respect to their diameters. Based on Fig. 5, we resolved three different size groups: $0-30 \mathrm{~km}, 30-60 \mathrm{~km}$ and $>60 \mathrm{~km}$.

The latitude distribution for asteroids with $D>60 \mathrm{~km}$ (Fig. 6a) is close to the uniform distribution for latitudes lower than $11^{\circ}$ and for larger latitudes it exhibits an evident excess of prograde rotators. This is in agreement with theoretical arguments presented by Davis et al. (1989) and recently by Johansen \& Lacerda (2010). On the other hand, the latitude distribution for asteroids with $D<30 \mathrm{~km}$ (Fig. 6e) exhibits a strong depopulation of pole vectors close to the ecliptic plane (i.e. small absolute values of latitudes $\beta$ ). The few asteroids with small latitudes have diameters $D>25 \mathrm{~km}$. The latitude distribution for asteroids with intermediate diameters of 30-60 km (Fig. 6c) is also somewhat clustered towards higher latitudes but the bins for small latitudes are more populated. Therefor, it is probably a transition region between the two distinct distributions.

It is evident that the depopulation concerns mainly objects with diameters $D \lesssim 30 \mathrm{~km}$ (the distribution for the intermediate size sample shows that the limit is probably $\sim 50 \mathrm{~km}$ ). This size roughly corresponds to the value, when the YORP effect starts to act and hence it is a natural candidate for a physical explanation. It is known from previous studies (Pravec \& Harris 2000; Rubincam 2000) that the YORP effect is significantly altering the periods and also spin vectors of these objects on a timescale shorter than the typical collisional lifetime of these objects (timescales are discussed in more details in Sect. 5). In Fig. 7, we show the relation between the spin rate and the latitude for the small $(D<30 \mathrm{~km})$ and large $(D>60 \mathrm{~km})$ groups of
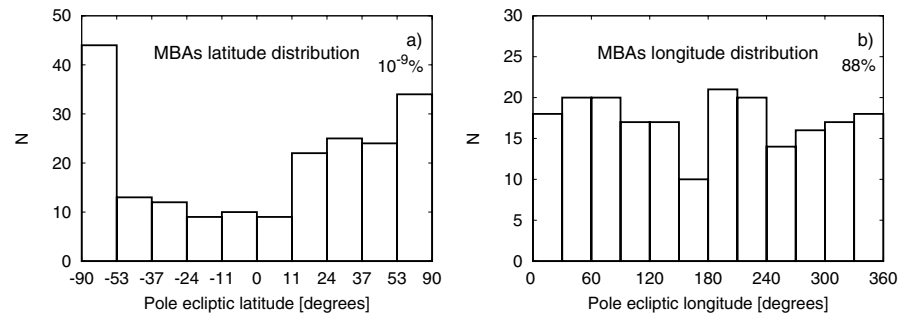

Fig. 4. a) Shows the ecliptic latitude distribution of all MBAs in our sample except the Koronis family members. The width of bins in latitude $\beta$ corresponds to similar surfaces on the $(\lambda, \beta)$-sphere, so the bins are equidistant in $\sin \beta$. In $\mathbf{b})$, the longitude distribution of all MBAs is plotted, again except the Koronis family members. The longitude bins are equidistant in $\lambda$. In the top right corners, there are the probability values of the $\chi^{2}$-tests (that the observed distributions are randomly drown from a uniform distribution, see Table 2).

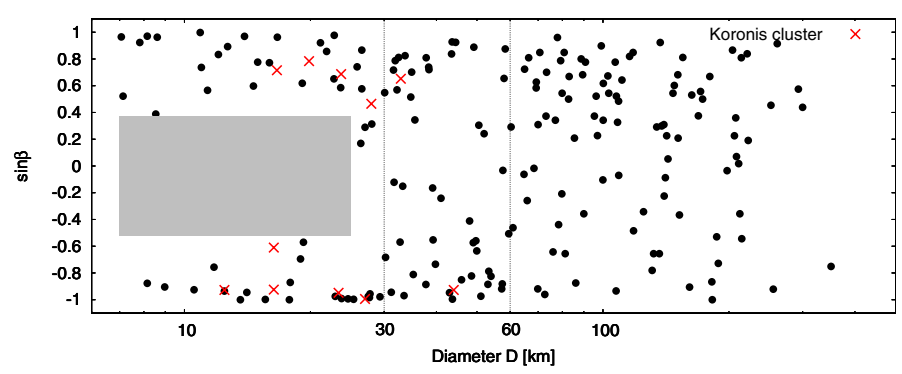

Fig. 5. Dependence of the ecliptic latitude $\beta$ (plotted as $\sin \beta$ ) of the pole direction on the models diameter $D$. The gray box shows the gap of small latitudes for asteroids with $D<30 \mathrm{~km}$. Members of the Koronis cluster are plotted with crosses.

asteroids. In concert with YORP theory, the spin up and spin down and the simultaneous evolution of the latitudes towards higher absolute values are evident in the small asteroid sample. 

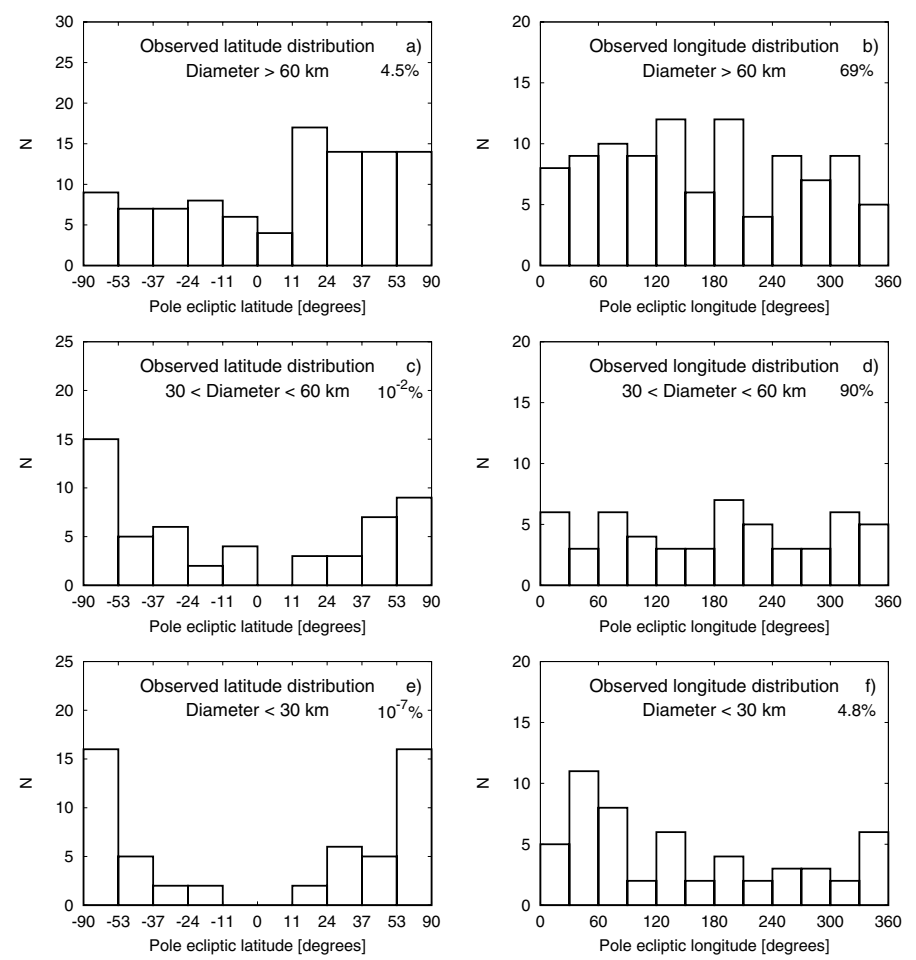

Fig. 6. Histograms showing the observed latitude and longitude distributions of MBAs (except Koronis cluster members) for different size ranges. a) Shows the latitude distribution for asteroids with diameters larger than $60 \mathrm{~km}, \mathbf{c}$ ) for asteroids with diameters in the range of 30-60 km, e) for asteroids with diameters smaller than $30 \mathrm{~km}$, and similarly b), d) and f) for longitudes. The width of bins in the latitude $\beta$ and longitude lambda $\lambda$ corresponds to similar surfaces on the $(\lambda, \beta)$-sphere, so the bins are equidistant in $\sin \beta$ and $\lambda$. In the top right corners, there are the probability values of the $\chi^{2}$-tests (that the observed distributions are randomly drown from a uniform distribution, see Table 2).
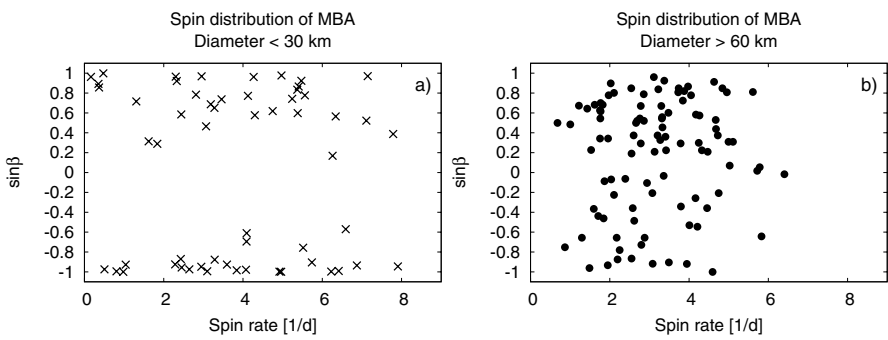

Fig. 7. Dependence of the ecliptic latitude $\beta$ (plotted as $\sin \beta$ ) of the pole direction on the models spin rate, a) for asteroids with diameter $D<30 \mathrm{~km}$ and $\mathbf{b}$ ) for asteroids with diameter $D>60 \mathrm{~km}$.

Note that observed latitude and longitude distributions can be biased by the convex inversion method and, therefore, this bias should be taken into consideration. In general, models for asteroids with higher amplitudes are more often successfully derived than those asteroids with lower amplitudes. This is because, while the accuracy of the sparse photometry in both cases is roughly the same, the signal-to-noise ratio is significantly better for higher amplitude lightcurves. If we assume two bodies with the same shapes and orbits and different ecliptic latitudes of the poles, the body with higher absolute value of ecliptic latitude usually has a higher amplitude. This effect is numerically investigated in Sect. 4.3.

Skoglöv \& Erikson (2002) discussed the role of orbital inclination in the observed distribution of latitudes. No indications of this effect were found in the asteroids sample of
Table 2. Test of the hypothesis that the observed pole distributions and de-biased latitude distribution are drown from uniform distributions (a $\chi^{2}$-test).

\begin{tabular}{lrrrrrr}
\hline \hline & \multicolumn{2}{c}{$\beta$} & \multicolumn{2}{c}{$\lambda$} & \multicolumn{2}{c}{$\beta_{\text {deb }}$} \\
& \multicolumn{2}{c}{$N=9$} & \multicolumn{2}{c}{$N=11$} & \multicolumn{2}{c}{$N=9$} \\
\hline Diameter & $\chi^{2}$ & $\%$ & $\chi^{2}$ & $\%$ & $\chi^{2}$ & $\%$ \\
all & 63 & $10^{-9}$ & 5.9 & 88 & 39 & $10^{-3}$ \\
$>60 \mathrm{~km}$ & 17 & 4.5 & 8.2 & 69 & 17 & 4.7 \\
$30-60 \mathrm{~km}$ & 30 & $10^{-2}$ & 5.6 & 90 & 18 & 2.9 \\
$<30 \mathrm{~km}$ & 59 & $10^{-7}$ & 20 & 4.8 & 37 & $10^{-3}$ \\
\hline
\end{tabular}

Notes. $N$ is the degree of freedom.

Kryszczyńska et al. (2007). We also did not find any indications of such correlation in our sample and so we conclude that orbital inclination does not affect the observed distribution of latitudes.

We are not aware of any other physical effects in the main belt that could explain the non-uniform observed latitude distribution of small asteroids $(D<30 \mathrm{~km})$. Collisions are believed to produce uniform spin distributions and close encounters with planets are common only among NEAs.

There are many additional selection effects that influence the properties of derived models, e.g. the role of amplitude, orbit, time, accuracy and geometry of observations, among others. The significance of this bias is unknown and cannot be easily determined. The main problem here is that for almost every asteroid, the photometric data are from different observers with a different number of measurements, quality, and purpose. The significance of this effect can be determined only from a comparison of models derived from real and synthetic data of known properties. This may be possible in a few years when the photometry from the Pan-STARRS is available, but not now. In the meantime, the role of the selection effects seems to be small and does not significantly affect, for example, the latitude distribution.

The longitude distributions of the MBAs are plotted in Figs. $4 \mathrm{~b}$ and $6 \mathrm{~b}, \mathrm{~d}, \mathrm{f}$. They are, contrary to the latitude distributions, without any statistically significant features and have very close to uniform distributions. The only exception are the asteroids with $D<30 \mathrm{~km}$, but the excess appears to be just a random coincidence than a result of some physical process.

In all cases, we tested a hypothesis that the observed distribution of latitudes or longitudes is uniform (using a $\chi^{2}$-test ${ }^{9}$ ). The computed chi-squares and corresponding probabilities are listed in Table 2. Higher $\chi^{2}$-values and lower probabilities mean that the supposed hypothesis "the observed distribution is uniform" does not fit the observed data. If we assume a probability of $5 \%$ or lower as statistically significant, we can say that the latitude distributions for the whole sample and for asteroids with $D<30 \mathrm{~km}$ and $30<D<60 \mathrm{~km}$ do not agree with a uniform distribution. On the other hand, all longitude distributions are consistent with uniform distributions. Latitude distribution for the MBAs with diameters $D>60 \mathrm{~km}$ also disagrees with the uniform distribution; this is because of the excess of prograde rotators.

The overall view on the model positions within the main belt of asteroids, together with their estimated total drifts and the information about whether they are prograde or retrograde rotators (Fig. 3), shows behavior consistent with the Yarkovsky/YORP theory: there is an asymmetry of prograde and retrograde rotators near the main resonances and prograde aster-

9 The results based on the $\chi^{2}$-test are also in agreement with the Kolmogorov-Smirnov test. 
oids drift outwards from the Sun and can reach the resonance only from the left. On the other hand, retrograde rotators drift the opposite direction and depopulate the zone left of the resonance because the resonance prevents the entry of new retrograde asteroids. This creates an excess of prograde rotators. The same mechanism works also in the zone right of the resonance, except, in this case, only an excess of retrograde asteroids is now created. This effect is obvious in the neighborhood of the $v_{6}$ and $3: 1$ resonances. The total drift from an asteroid's original location during the collisional lifetime computed with Eq. (7) are inversely proportional to the size of the asteroids. Larger asteroids $(D \gtrsim 50 \mathrm{~km})$ do not drift significantly while smaller asteroids frequently drift $\pm 0.05 \mathrm{AU}$. Note that small-sized asteroids are found mainly in the inner or middle part of the main belt (due to selection effect; they have high albedos and/or are closer to the Earth). Asteroids drifting through the resonances during their collisional lifetime are interesting from the point of the dynamical evolution. These asteroids were either recently collisionallyaffected or their shape models are wrong. There seems to be three such models, two near the 3:1 resonance and one near the resonance 5:2.

\subsection{The Koronis family members}

The analysis of rotational state solutions for ten members of the Koronis asteroid family revealed a clustered distribution of their spin vectors (Slivan 2002; Slivan et al. 2003). This spin distribution was later explained by Vokrouhlický et al. (2003) as the result of the thermal torques and spin-orbital resonances that modify the spin states over time of 2-3 Gyr. The modeling suggested an existence of two groups of asteroids: (a) low-obliquity retrograde objects with rotational periods $P<5$ h or $P>13 \mathrm{~h}$, and (b) prograde rotators with periods $4<P<7$ h that became trapped in a spin-orbit resonance with secular frequency $\mathrm{s}_{6}$ and thus have similar spin obliquities $\left(42-51^{\circ}\right)$ and also similar ecliptic longitudes in the range of $\left(24-73^{\circ}\right)$ and $\left(204-259^{\circ}\right)$. All ten members of the Koronis family studied by Slivan (2002) and Slivan et al. (2003) had the expected properties: periods for prograde rotators were shifted only to higher values of 7-10 h. Slivan et al. (2009) published spin state solutions for another four members of Koronis family. Only the solution for (253) Dresda was not in agreement with the theoretical expectation.

Here, we present three new models of asteroids belonging to the Koronis family: (832) Karin, (1482) Sebastiana, and (1635) Bohrmann, along with two partial models for (1350) Rosselina and (1389) Onnie. Only the spin state solutions for Bohrmann and Onnie fit the theoretical expectations. Rotational parameters for Karin $\left(P=18.3512 \mathrm{~h}, \lambda=242^{\circ}, \beta=46^{\circ}\right)$ are outside both groups. Asteroids Sebastiana and Rosselina are low-obliquity retrograde rotators, but their periods $(10.49 \mathrm{~h}$ for Sebastiana and $8.14 \mathrm{~h}$ for Rosselina) are in the middle of the "wrong" range of $P=5-13 \mathrm{~h}$. Karin is the namesake and largest member of a small and young ( 5.8 My, Nesvorný \& Bottke 2004) collisional family that is confined within the larger Koronis family. The spin state of Karin was thus likely affected during this catastrophic event and changed to a random state that disagrees with the clustered distribution.

We are not able to give a satisfactory explanation for the peculiar spin state solutions for Sebastiana and Rosselina. Nevertheless, we are aware of two possible scenarios: (i) the initial rotational state and shape did not allow being captured in the resonance or (ii) the objects were randomly reoriented by non-catastrophic collisions. The timescales of such collisions (given by Eq. (7)) are for Sebastiana $\tau_{\text {reor }} \sim 7.5$ Gyr and for Rosselina $\tau_{\text {reor }} \sim 14.7$ Gyr. This leads to the probability of a collision during the Koronis cluster lifetime (estimated to $\sim 2.5$ Gyr, Bottke et al. 2001) 1/3 for Sebastiana and $\sim 1 / 6$ for Rosselina, respectively, which means that random collisional reorientation of the spin axis is likely for at least a few of 19 asteroids in the Koronis cluster with known spin state solutions (most of them have $\tau_{\text {reor }} \lesssim 20 \mathrm{Gyr}$ ).

\subsection{Biases of the LI method}

We developed a numerical algorithm to estimate the selection effect of the lightcurve inversion method and used this approach to de-bias the observed distribution of asteroid's pole directions. The algorithm was as follows:

1. for a model with a known shape, we randomly generated a new pole direction (while the overall distribution of poles was isotropic);

2. for each shape with a new rotational state but with the period unchanged, we computed synthetic lightcurves for the same epochs as observed ones;

3. to each data point $i$, we added the corresponding noise $\delta_{i}$ given by:

$\delta_{i}=\frac{L_{i}^{\mathrm{obs}}-L_{i}^{\mathrm{mod}}}{L_{i}^{\mathrm{mod}}}$,

where $L_{i}^{\mathrm{obs}}$ is $i$ th brightness observed and $L_{i}^{\mathrm{mod}}$ is $i$ th brightness computed, both for the original model. This gave us synthetic lightcurve data equivalent to the original observed data, but for a new pole direction;

4. finally, we performed a lightcurve inversion the same way as with the actual data and tried to derive a model;

5. we repeated steps $1-4$ for 50 random poles for each asteroid model.

In this simulation, we used 80 models derived from combined dense and sparse data sets and 89 models from the DAMIT.

For each successfully derived model we have the generated pole direction $\left(\lambda_{\mathrm{gen}}, \beta_{\mathrm{gen}}\right)$ and period $P_{\mathrm{gen}}$, and also the computed pole and period: $\lambda_{\text {comp }}, \beta_{\text {comp }}, P_{\text {comp }}$. We computed the angular differences between the generated and derived ecliptic latitude and longitude of the pole direction: $\beta_{\text {comp }}-\beta_{\text {gen }}$ and $\left(\lambda_{\text {comp }}-\lambda_{\text {gen }}\right) \cos \beta_{\text {gen }}$ (the $\cos \beta_{\text {gen }}$ factor is used for the correction of the different distances of meridians near the equator and poles). In Fig. 8, we show the histograms of these differences in a) ecliptic latitudes and b) ecliptic longitudes. We assumed that the histograms can be described by a normal distribution and we computed the mean and the standard deviation $(\mu ; \sigma)$. We found values of $(-0.2 ; 10.2)$ for latitudes and $(-0.2 ; 5.2)$ for longitudes. The standard deviation $\sigma$ is directly related to the typical uncertainty that we can expect in pole determination by the lightcurve inversion method, which is $\sim 5^{\circ} / \cos \beta$ in $\lambda$ and $\sim 10^{\circ}$ in $\beta$.

In Fig. 9a, we constructed a histogram of the latitude distribution for all successful models. The bins in $\beta$ were again equally spaced in $\sin \beta$. The latitude distribution of all generated models was not exactly uniform, the amount of latitudes in bins slightly differed. To remove this effect, we divided the latitude distribution of successfully derived models by the latitude distribution of all generated models normalized to unity. This correction was also applied to latitude distributions in Figs. 10a, c, e. It is obvious that the LI method is more efficient for asteroids with higher $|\beta|$. The amount of successfully derived models with $|\beta| \sim 0^{\circ}$ is about $30 \%$ lower than with $|\beta|>53^{\circ}$. 

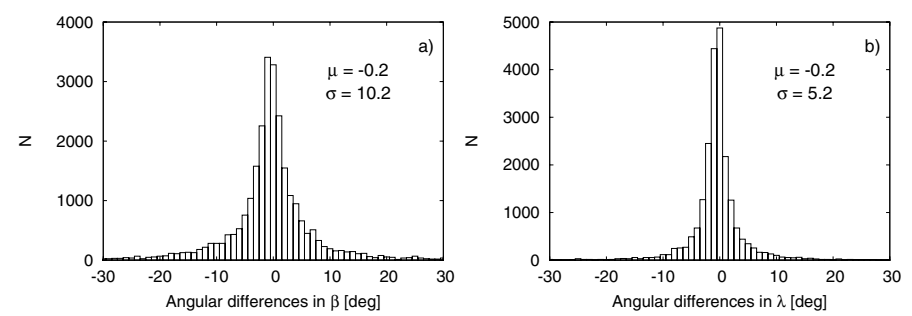

Fig. 8. Histograms of the angular differences between the generated and derived ecliptic latitudes and longitudes of the pole directions for all successfully derived synthetic models: a) $\beta_{\text {comp }}-\beta_{\text {gen }}$, where $\beta_{\text {gen }}$ and $\beta_{\text {comp }}$ are generated and computed ecliptic latitudes and similarly b) $\left(\lambda_{\text {comp }}-\lambda_{\text {gen }}\right) \cos \beta_{\text {gen }}$ for ecliptic longitudes.
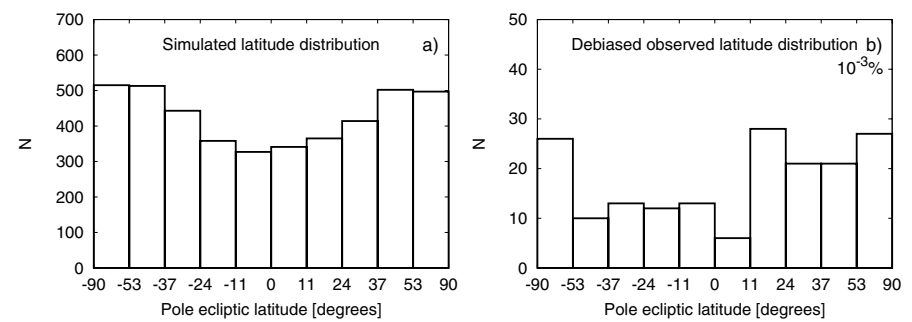

Fig. 9. a) Shows the simulated ecliptic latitude distribution of all successfully derived models. In b), the de-biased observed latitude distribution is plotted. The bins are equidistant in $\sin \beta$. In the top right corner, there is the probability value of the $\chi^{2}$-test (that the observed distribution is drown from a uniform one, see Table 2).

In Figs. 10a, c, e, we constructed the histograms of latitude distributions for successfully derived models and distinguished three size ranges. All three plots look very similar, except that with decreasing size, the ratio between models with $|\beta| \sim 0^{\circ}$ and $|\beta|>53^{\circ}$ goes down. This ratio is $\sim 75 \%$ for $D>60 \mathrm{~km}, \sim 65 \%$ for $30<D<60 \mathrm{~km}$ and $\sim 60 \%$ for $D<30 \mathrm{~km}$.

The histograms in Figs. 9a and 10a, c, e define the bias in latitude of the LI method and can be used for de-biasing the observed latitude distributions presented in Figs. 4a and 6a, c, e. The de-biased histograms of latitudes are plotted in Figs. 9b and $10 \mathrm{~b}, \mathrm{~d}, \mathrm{f}$. The histograms changed only slightly and the conclusions from Sect. 4.1 are still valid, i.e., the latitude distribution differs significantly from a uniform distribution, and especially so for $D<30 \mathrm{~km}$. The distribution of latitudes for asteroids with $D>60$ has an evident excess of prograde rotators while the distribution for a subsample with $30 \mathrm{~km}<D<60 \mathrm{~km}$ shows an enrichment of asteroids with large latitudes $\left(|\beta|>53^{\circ}\right)$. Other bins have similar populations. We also performed a $\chi^{2}$-test in the same way as for the observed distributions (see Table 2, Cols. 6 and 7).

We did not find any significant correlation between the ecliptic longitude and the efficiency of the model determination.

\section{A theoretical model of the latitude distribution}

In order to understand observations of main-belt asteroids, namely the de-biased distribution of their ecliptic latitudes $\beta$ (Fig. 9b), we constructed a simple model for spin evolution that contains the following processes: (i) the YORP effect, i.e., torques arising from the emission of thermal radiation, (ii) random reorientations induced by non-catastrophic collisions, and (iii) mass shedding after a critical rotational frequency is reached.

On the other hand, we did not include gravitational torques of the Sun, spin-orbital resonances, damping (dissipation of
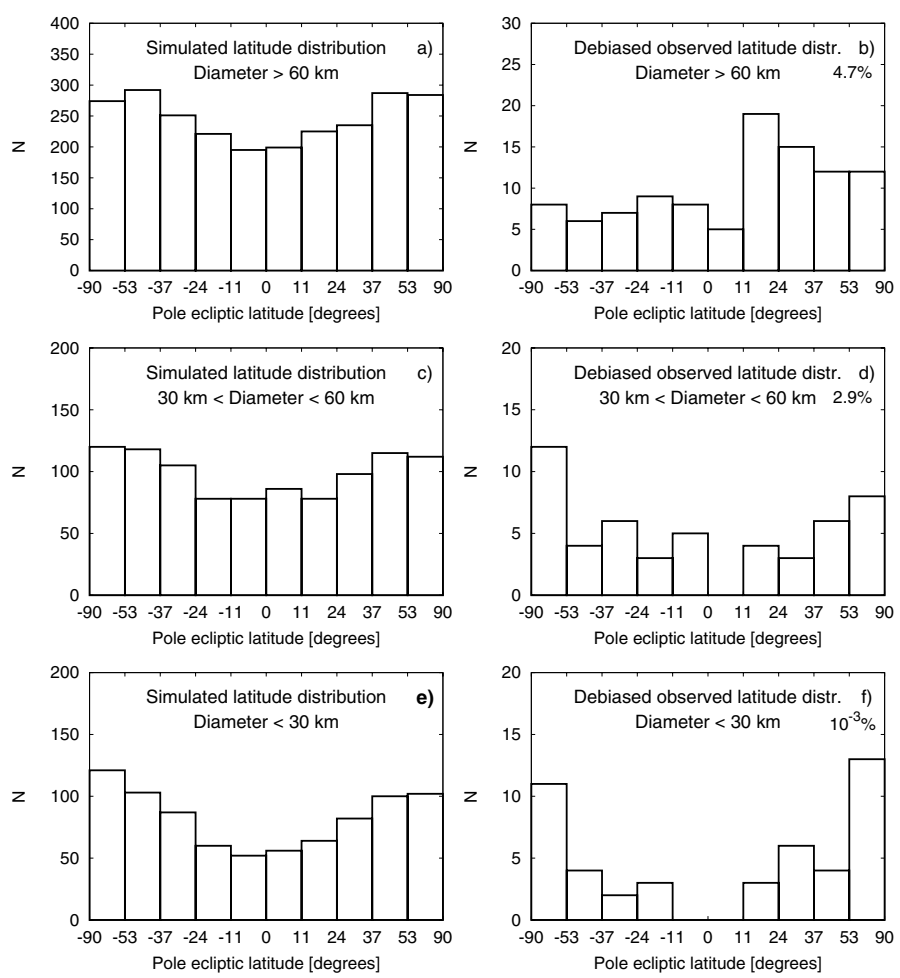

Fig. 10. Histograms showing the simulated and corrected (de-biased) observed latitude distributions of MBAs for different size groups, excluding the Koronis cluster members. a) Shows the simulated latitude distribution for asteroids with the diameters larger than $60 \mathrm{~km}$, c) for asteroids with the size in range of 30-60 km, e) for asteroids with diameters smaller than $30 \mathrm{~km}$. Similarly b), d) and f) show the observed latitude distributions corrected by the bias of the LI method. The bins are equidistant in $\sin \beta$. In the top right corners, there are the probability values of the $\chi^{2}$-tests (that the observed distributions are drown from a uniform distribution, see Table 2).

rotational energy), or tumbling. Even though individual asteroids may be substantially affected by these processes, our model is for a large statistical sample of asteroids and the effect on the overall latitude distribution is assumed to be only minor. For example, gravitational torques and spin-orbital resonances usually cause large oscillations of $\beta$ for prograde-rotating asteroids, but they remain bound to a certain interval (Vokrouhlický et al. 2006). Moreover, we tried to account for these (rather random) oscillations in our model as well (see below).

Our sample of 220 asteroids was the same as the observed sample discussed in Sect. 4. This means that the orbits and sizes correspond to real asteroids. The model for spin evolution was similar to that of Brož et al. (2011), where it was used for studies of the long-term evolution of asteroid families. We assumed the following relations for the rate of the angular velocity $\omega$ and the obliquity $\epsilon$ due to the YORP effect

$$
\begin{aligned}
& \frac{\mathrm{d} \omega}{\mathrm{d} t}=f_{i}(\epsilon), \quad i=1 \ldots 200, \\
& \frac{\mathrm{d} \epsilon}{\mathrm{d} t}=\frac{g_{i}(\epsilon)}{\omega},
\end{aligned}
$$

where $f$ - and $g$-functions were given by Čapek \& Vokrouhlický (2004) for a set of 200 shapes with mean radius $R_{0}=1 \mathrm{~km}$, bulk density $\rho_{0}=2500 \mathrm{~kg} / \mathrm{m}^{3}$, located on a circular orbit with semimajor axis $a_{0}=2.5 \mathrm{AU}$. We assigned one of the artificial shapes 

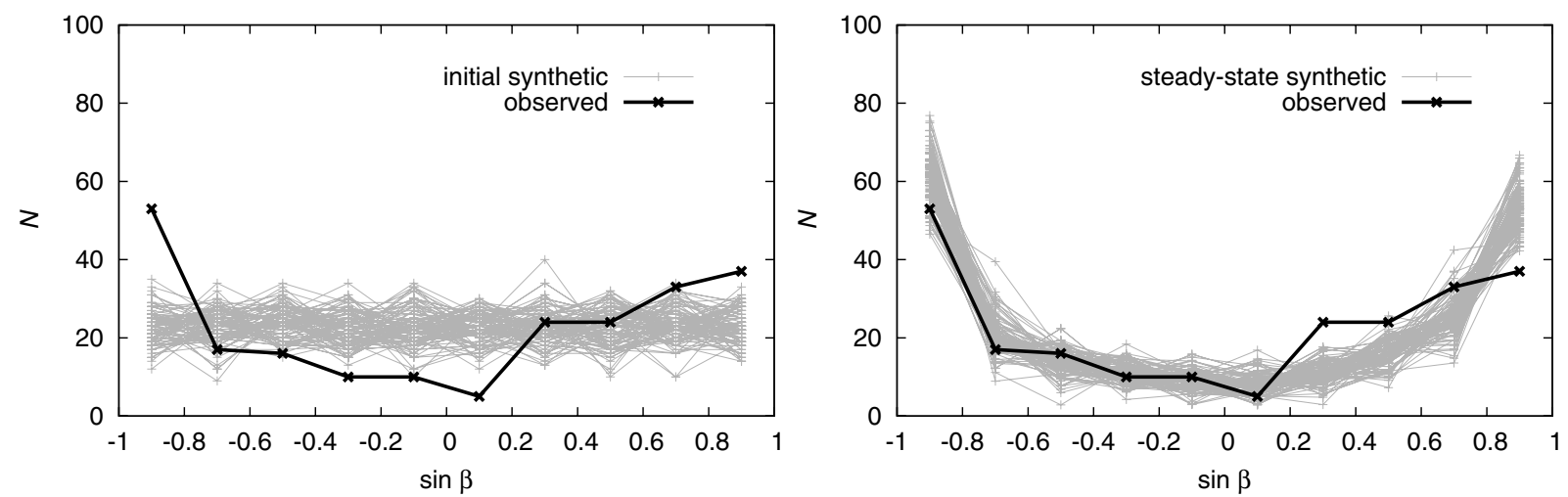

Fig. 11. Left panel: the distribution of the ecliptic latitudes $\beta$ for the observed asteroids (thick line) and 100 synthetic samples (generated with a different random seed) at time $t=0$ (thin lines). Right panel: steady-state synthetic latitude distributions at time $t=4 \mathrm{Gyr}$ (thin lines), evolved by the YORP effect, collisions, mass shedding and spin-orbit resonances. We also applied an observational bias to it. The steady state was reached already within $\simeq 1 \mathrm{Gyr}$.

(denoted by the index $i$ ) randomly to each individual asteroid ${ }^{10}$. We had only to scale the $f$ - and $g$-functions by a factor

$c=c_{\mathrm{YORP}}\left(\frac{a}{a_{0}}\right)^{-2}\left(\frac{R}{R_{0}}\right)^{-2}\left(\frac{\rho_{\text {bulk }}}{\rho_{0}}\right)^{-1}$,

where $a, R, \rho_{\text {bulk }}$ are semi-major axis, radius, and density of the simulated body, respectively, and $c_{\text {YORP }}$ is a free scaling parameter, which can account for an additional uncertainty of the YORP model. Because the values of $f$ 's and $g$ 's were computed for only a limited set of obliquities (with a step $\Delta \epsilon=30^{\circ}$ ) we used interpolation by Hermite polynomials (Hill 1982) of the data in Čapek \& Vokrouhlický (2004) to obtain a smooth analytical functions for $f_{i}(\epsilon)$ and $g_{i}(\epsilon)$.

When the angular velocity approached a critical value (i.e., the gravity was equal to the centrifugal force)

$\omega_{\text {crit }}=\sqrt{\frac{4}{3} \pi G \rho_{\text {bulk }}}$

we assumed a mass shedding event. We kept the orientation of the spin axis and the sense of rotation but reset the orbital pe$\operatorname{riod} P=2 \pi / \omega$ to a random value from the interval $\left(P_{1}, P_{2}\right)=$ $(2.5,9) \mathrm{h}$. We also altered the assigned shape since any change of shape can produce a different YORP effect. We did not change the mass, however.

The differential Eqs. (3) and (4) were integrated numerically by a simple Euler integrator. The usual time step was $\Delta t=1000 \mathrm{yr}$. The time scale of the spin axis evolution for small bodies $(D \simeq 10 \mathrm{~km})$ is $\tau_{\text {YORP }} \simeq 500$ Myr. After $\simeq 3$ times $\tau_{\text {YORP }}$ most of these bodies have spin axes perpendicular to the ecliptic.

We also included a Monte-Carlo model for spin axis reorientations caused by collisions ${ }^{11}$. We used an estimate of the time

\footnotetext{
10 We did not use the convex-hull shapes derived in this work for two reasons: (i) the two samples of shapes are believed to be statistically equivalent and it is thus not necessary to compute the YORP torques again; (ii) the YORP effect seems sensitive to small-scale surface structure (Scheeres \& Mirrahimi 2007) which cannot be caught by our shape model. Nevertheless, the YORP torque remains of the same order, so the random assignment of shapes seems reasonable.

11 Collisional disruptions are not important, since we are only interested in the steady state. We can imagine that whenever an asteroid from our sample is disrupted, another one with a randomly oriented spin axis is created by a disruption of a larger body.
}

scale by Farinella et al. (1998)

$\tau_{\text {reor }}=B\left(\frac{\omega}{\omega_{0}}\right)^{\beta_{1}}\left(\frac{D}{D_{0}}\right)^{\beta_{2}}$,

where $B=84.5 \mathrm{kyr}, \beta_{1}=5 / 6, \beta_{2}=4 / 3, D_{0}=2 \mathrm{~m}$ and $\omega_{0}$ corresponds to period $P=5 \mathrm{~h}$. These values are characteristic for the main belt. After a collision, we reset the spin axis periods to random values, using the interval $\left(P_{1}^{\prime}, P_{2}^{\prime}\right)=(2.5,9) \mathrm{h}$ for the period. Since the time scale is $\tau_{\text {reor }} \simeq 3$ Gyr for the smallest $(D \simeq 5 \mathrm{~km})$ bodies, reorientations are only of minor importance. However, note that the probability of the reorientation is enhanced when the YORP effect drives the angular velocity $\omega$ close to zero.

There were several free parameters in our model: the $c_{\text {YORP }}$ parameter, thermal conductivity $K$, bulk density $\rho_{\text {bulk }}$, initial distribution of $\beta$ and initial distribution of $\omega$.

Our aim was to start with a simple $\beta$ - and $\omega$-distribution, wait until a steady state was reached, and then compare the resulting synthetic to observed latitude distributions. We applied an observational bias derived in Sect. 4.3 to the synthetic distribution.

We partly accounted for spin-orbital resonances acting on prograde asteroids by adding a sinusoidal oscillations to $\beta$ with a random phase and an amplitude $\simeq 40^{\circ}$, which are typically induced by resonances. This procedure naturally decreased the right-most bin $(\sin \beta=(0.8,1))$ of the synthetic distribution and increased the next bin $(\sin \beta=(0.6,0.8))$.

We started with reasonable parameters of $c_{\text {YORP }}=0.33, K=$ $10^{-2} \mathrm{~W} / \mathrm{K} / \mathrm{m}, \rho_{\text {bulk }}=2500 \mathrm{~kg} / \mathrm{m}^{3}$, a Maxwellian distribution of $\omega$, a uniform distribution of $\sin \beta$ (i.e., an isotropic distribution of spin axes). We ran 100 such simulations with different random seeds. A steady state was reached within $\simeq 1$ Gyr. The resulting latitude distributions are shown in Figs. 11 and 12.

From these it can be seen that: (i) the observed distribution of $\beta$ for small asteroids seems compatible with our model; the YORP effect is capable of creating such an uneven distribution and (ii) there is a discrepancy for large asteroids (especially in bins $\sin \beta \in(-1,-0.8)$ and $(0.2,0.4))$, which can be explained as a preference for prograde rotators in the primordial population (see Davis et al. 1989; Johansen \& Lacerda 2010). The results regarding the spin rates agree with Pravec et al. (2008), so we do not repeat the discussion here.

We also tested the sensitivity of our results with respect to the free parameters. The thermal conductivity did not seem important (we tested $K=10^{-3} \mathrm{~W} / \mathrm{K} / \mathrm{m}$ ). A simulation with $c_{\text {YORP }}=0.66, \rho=1300 \mathrm{~kg} / \mathrm{m}^{3}$, and a uniform distribution of 


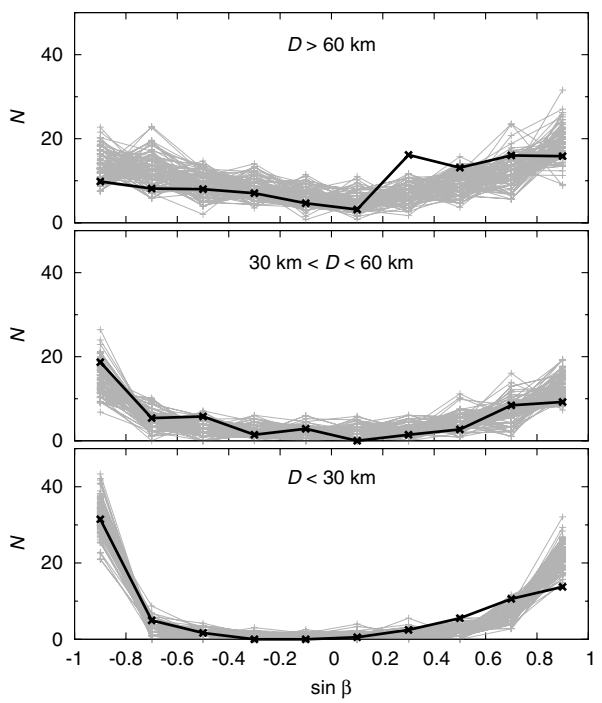

Fig. 12. Steady-state synthetic latitude distributions for three different size ranges: $D>60 \mathrm{~km}$ (top), $30 \mathrm{~km}<D<60 \mathrm{~km}$ (middle) and $D<$ $30 \mathrm{~km}$ (bottom). The synthetic distributions are plotted by thin gray lines while the observed distribution by a thick black line.

orbital periods $P \propto 1 / \omega$ produced almost the same resulting latitude distribution. Nevertheless, a value of $c_{\text {YORP }}=1.00$ seems too high because the extreme bins of the $\beta$-distribution were overpopulated $^{12}$. There is only a weak dependence of our results on the period ranges that we used for resetting the orbital period after a mass-shedding event $\left(P_{1}, P_{2}\right)$ and collision $\left(P_{1}^{\prime}, P_{2}^{\prime}\right)$. As would be expected, the values $P_{1}, P_{2}, P_{1}^{\prime}, P_{2}^{\prime}$ significantly affect the period distribution. The relatively weak dependence on the free parameters likely stems from the fact that we presume the steady-state. Even though the free parameters change, e.g., the strength of the YORP effect and the evolution of spins is slower and/or faster, after reaching a steady state, the basic characteristics of the latitude distribution remain similar. The observed $\beta$ distribution of small asteroids $(D<30 \mathrm{~km})$ cannot be explained by our simulation without accounting the YORP effect.

\section{Conclusions}

The results of this paper can be summarized as follows.

We used combined dense and sparse data to derive new asteroid shape models. We systematically gathered and processed all available sparse photometry from astrometric surveys and employed valuable data from seven observatories (see Table 1) in lightcurve inversion.

We derived 80 new unique models of asteroids, from which 16 are based only on sparse data. We also present 30 partial models with accurate rotational periods and estimated ecliptic latitudes of the pole directions and 18 updated solutions based on new data for asteroids already included in DAMIT.

In the future, quality sparse data sets will be produced by allsky surveys such as Pan-STARRS, the Large Synoptic Survey Telescope (LSST), and the Gaia satellite. When these data are available, we will be able to apply the same methods in order to derive many more new asteroid models. These surveys will have

\footnotetext{
12 As an alternative hypothesis, we assumed the spin axis evolution without a YORP effect $\left(c_{\text {YORP }}=0\right)$. In this case, the initial $\beta$ distributions (Fig. 11, left panel, thin lines) do not change significantly in time.
}

one advantage over dense data: their selection effects (e.g., with respect to the orbit) will be known. This will allow us to make a more accurate analysis of the asteroid population.

As expected, the observed ecliptic longitude distribution of asteroid spin vector is independent of diameter and is compatible with a uniform distribution. Unlike the latitude distribution, the observed ecliptic longitude distribution is not significantly biased by the LI method. However, the effect of the LI bias is only minor and the global features of the observed latitude distribution do not change. The observed (and de-biased) latitude distribution for asteroids with diameters $D>60 \mathrm{~km}$ shows an excess of prograde rotators in the latitude interval $\left(11^{\circ}, 90^{\circ}\right)$. This excess is probably primordial. On the other hand, the latitude distributions for the entire sample and in particular for asteroids with $D<30 \mathrm{~km}$, is strongly anisotropic.

The dynamical evolution of asteroid spins seems to be dominated by the YORP effect and also by collisions and mass shedding for asteroids with diameters $D \lesssim 30 \mathrm{~km}$. We calculate that YORP (with a small contribution for the LI method's bias) is capable of producing the observed depopulation of spin vectors for small asteroids.

We are not yet able to study small asteroids in individual families (small bodies at the outskirts of a family should have extreme spins); this is an aim of future work.

Acknowledgements. The work of J.H. has been supported by the grant GA UK 134710 of the Grant agency of the Charles University, by the project SVV 261301 of the Charles University in Prague and by the grant GACR 205/08/H005 of the Czech grant agency. The work of J.H. and J.D. has been supported by the grant GACR 209/10/0537 of the Czech grant agency, the work of J.D. and M.B. by the Research Program MSM0021620860 of the Czech Ministry of Education and the work of M.B. has been also supported by the Grant Agency of the Czech Republic (grant 205/08/P196). The calculations were performed on the computational cluster Tiger at the Astronomical Institute of Charles University in Prague (http://sirrah.troja.mff.cuni.cz/tiger).

We thank Brian D. Warner for significantly improving the linguistic quality of this paper and David Čapek for sending us the YORP effect data in an electronic form.

\section{References}

Bottke, W. F., Vokrouhlický, D., Brož, M., Nesvorný, D., \& Morbidelli, A. 2001, Science, 294, 1693

Bottke, J. W. F., Vokrouhlický, D., Rubincam, D. P., \& Nesvorný, D. 2006, Ann. Rev. Earth Planet. Sci., 34, 157

Brož, M., Vokrouhlický, D., Morbidelli, A., Nesvorný, D., \& Bottke, W. F. 2011, MNRAS, in press

Čapek, D., \& Vokrouhlický, D. 2004, Icarus, 172, 526

Davis, D. R., Weidenschilling, S. J., Farinella, P., Paolicchi, P., \& Binzel, R. P. 1989, in Asteroids II, ed. R. P. Binzel, T. Gehrels, \& M. S. Matthews, 805

Dobrovolskis, A. R. 1996, Icarus, 124, 698

Drummond, J. D., Weidenschilling, S. J., Chapman, C. R., \& Davis, D. R. 1988, Icarus, 76,19

Drummond, J. D., Weidenschilling, S. J., Chapman, C. R., \& Davis, D. R. 1991, Icarus, 89,44

Ďurech, J., Grav, T., Jedicke, R., Denneau, L., \& Kaasalainen, M. 2005, Earth Moon and Planets, 97, 179

Ďurech, J., Scheirich, P., Kaasalainen, M., et al. 2007, IAU Symp. 236, ed. G. B. Valsecchi, D. Vokrouhlický, \& A. Milani, 191

Durech, J., Kaasalainen, M., Warner, B. D., et al. 2009, A\&A, 493, 291

Durech, J., Sidorin, V., \& Kaasalainen, M. 2010, A\&A, 513, A46

Durech, J., Kaasalainen, M., Herald, D., et al. 2011, Icarus, accepted

Farinella, P., Vokrouhlicky, D., \& Hartmann, W. K. 1998, Icarus, 132, 378

Hill, G. 1982, Publ. Dom. Astrophys. Obs. Victoria BC, 16, 67

Ito, T., \& Yoshida, F. 2007, PASJ, 59, 269

Johansen, A., \& Lacerda, P. 2010, MNRAS, 404, 475

Kaasalainen, M. 2004, A\&A, 422, L39

Kaasalainen, M., \& Torppa, J. 2001, Icarus, 153, 24

Kaasalainen, M., Torppa, J., \& Muinonen, K. 2001, Icarus, 153, 37

Kaasalainen, M., Mottola, S., \& Fulchignoni, M. 2002, Asteroids III, 139

Kryszczyńska, A., La Spina, A., Paolicchi, P., et al. 2007, Icarus, 192, 223 
La Spina, A., Paolicchi, P., Kryszczyńska, A., \& Pravec, P. 2004, Nature, 428, 400

Lagerkvist, C., Barucci, M. A., Capria, M. T., et al. 1987, Asteroid photometric catalogue., ed. C.-I. Lagerkvist, M. A. Barucci, M. T. Capria, M. Fulchignoni, L. Guerriero, E. Perozzi, \& V. Zappala

Magnusson, P. 1986, Icarus, 68, 1

Magnusson, P. 1990, Icarus, 85, 229

Morbidelli, A., \& Vokrouhlický, D. 2003, Icarus, 163, 120

Nesvorný, D., \& Bottke, W. F. 2004, Icarus, 170, 324

Piironen, J., Lagerkvist, C., Torppa, J., Kaasalainen, M., \& Warner, B. 2001, in BAAS, 33, 1562

Pilcher, F. 2008, Minor Planet Bulletin, 35, 135

Pilcher, F. 2009, Minor Planet Bulletin, 36, 133

Pilcher, F. 2010, Minor Planet Bulletin, 37, 167

Pilcher, F., \& Cooney, J. W. R. 2006, Minor Planet Bulletin, 33, 13

Pilcher, F., \& Jardine, D. 2009, Minor Planet Bulletin, 36, 52

Pilcher, F., \& Oey, J. 2010, Minor Planet Bulletin, 37, 144

Pravec, P., \& Harris, A. W. 2000, Icarus, 148, 12

Pravec, P., Harris, A. W., \& Michalowski, T. 2002, Asteroids III, 113

Pravec, P., Harris, A. W., Vokrouhlický, D., et al. 2008, Icarus, 197, 497

Rubincam, D. P. 2000, Icarus, 148, 2

Scheeres, D. J., \& Mirrahimi, S. 2007, in BAAS, 38, 416

Skoglöv, E., \& Erikson, A. 2002, Icarus, 160, 24
Slivan, S. M. 2002, Nature, 419, 49

Slivan, S. M., \& Molnar, L. A. 2010, in BAAS, 42, 1036

Slivan, S. M., Binzel, R. P., Crespo da Silva, L. D., et al. 2003, Icarus, 162, 285

Slivan, S. M., Binzel, R. P., Kaasalainen, M., et al. 2009, Icarus, 200, 514

Stephens, R. D. 2003, Minor Planet Bulletin, 30, 46

Stephens, R. D. 2004, Minor Planet Bulletin, 31, 40

Stephens, R. D. 2005a, Minor Planet Bulletin, 32, 82

Stephens, R. D. 2005b, Minor Planet Bulletin, 32, 27

Stephens, R. D. 2005c, Minor Planet Bulletin, 32, 2

Stephens, R. D., \& Warner, B. D. 2004, Minor Planet Bulletin, 31, 3

Tedesco, E. F., Noah, P. V., Noah, M., \& Price, S. D. 2002, AJ, 123, 1056

Vokrouhlický, D., Nesvorný, D., \& Bottke, W. F. 2003, Nature, 425, 147

Vokrouhlický, D., Nesvorný, D., \& Bottke, W. F. 2006, Icarus, 184, 1

Warner, B. D. 2002, Minor Planet Bulletin, 29, 74

Warner, B. D. 2003, Minor Planet Bulletin, 30, 21

Warner, B. D. 2005a, Minor Planet Bulletin, 32, 54

Warner, B. D. 2005b, Minor Planet Bulletin, 32, 26

Warner, B. D. $2011 \mathrm{a}$, Minor Planet Bulletin, 38, 25

Warner, B. D. 2011b, Minor Planet Bulletin, 38, 52

Warner, B. D. 2011c, Minor Planet Bulletin, 38, 63

Warner, B. D., Harris, A. W., \& Pravec, P. 2009, Icarus, 202, 134

Yoshida, F., Dermawan, B., Ito, T., et al. 2004, PASJ, 56, 1105

Pages 13 to 16 are available in the electronic edition of the journal at http://www . aanda.org 
J. Hanuš et al.: A study of asteroid pole-latitude distribution

Table 3. List of new asteroid models derived from combined data sets or sparse data alone.

\begin{tabular}{|c|c|c|c|c|c|c|c|c|c|c|c|c|c|c|c|}
\hline & Asteroid & $\begin{array}{c}\lambda_{1} \\
{[\mathrm{deg}]}\end{array}$ & $\begin{array}{c}\beta_{1} \\
{[\mathrm{deg}]}\end{array}$ & $\begin{array}{c}\lambda_{2} \\
{[\mathrm{deg}]}\end{array}$ & $\begin{array}{c}\beta_{2} \\
{[\mathrm{deg}]}\end{array}$ & $\begin{array}{c}P \\
{[\mathrm{~h}]}\end{array}$ & $\overline{N_{\mathrm{lc}}}$ & 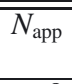 & $\overline{N_{689}}$ & $\overline{N_{699}}$ & 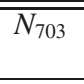 & $\overline{N_{\text {E12 }}}$ & 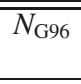 & $N_{950}$ & $\overline{\bar{N} N_{\text {Hip }}}$ \\
\hline 10 & Hygiea & 312 & -42 & 122 & -44 & 27.6591 & 23 & 9 & 263 & & & & & 405 & 50 \\
\hline 13 & Egeria & 44 & 21 & 238 & 11 & 7.04667 & 13 & 4 & 255 & & 74 & & & 203 & 34 \\
\hline 14 & Irene & 97 & -22 & 268 & -24 & 15.02991 & 20 & 8 & 250 & & & 48 & & 161 & 45 \\
\hline 37 & Fides & 270 & 19 & 89 & 27 & 7.33253 & 23 & 5 & 270 & & 61 & & & 135 & 31 \\
\hline 40 & Harmonia & 22 & 31 & 206 & 39 & 8.90848 & 19 & 6 & 210 & & 48 & & & 255 & 102 \\
\hline 42 & Isis & 106 & 40 & 302 & 28 & 13.58364 & 28 & 7 & 210 & & 36 & & & 128 & 51 \\
\hline 62 & Erato & 87 & 22 & 269 & 23 & 9.21819 & 1 & 1 & 164 & & 48 & & & & \\
\hline 68 & Leto & 103 & 43 & 290 & 23 & 14.84547 & 12 & 2 & 174 & & 85 & 30 & & 152 & \\
\hline 69 & Hesperia & 250 & 17 & 71 & -2 & 5.65534 & 35 & 7 & 222 & & 44 & 40 & & & \\
\hline 97 & Klotho & -1 & 30 & 161 & 40 & 35.2510 & 25 & 6 & 309 & & 31 & & & 202 & \\
\hline 119 & Althaea & 339 & -67 & 181 & -61 & 11.46514 & 4 & 2 & 149 & & 59 & & & 222 & \\
\hline 162 & Laurentia & 139 & 64 & 313 & 51 & 11.86917 & 4 & 2 & 166 & 31 & 40 & & & & \\
\hline 174 & Phaedra & 94 & 36 & 266 & 14 & 5.75025 & 2 & 1 & 173 & & 36 & & & & \\
\hline 188 & Menippe & 32 & 48 & 198 & 25 & 11.9765 & 4 & 1 & 145 & & 40 & & & & \\
\hline 258 & Tyche & 224 & -4 & 40 & -9 & 10.04008 & 10 & 2 & 162 & & 44 & & & & \\
\hline 264 & Libussa & 157 & 18 & 338 & -9 & 9.22794 & 19 & 3 & 129 & & 39 & 49 & & & \\
\hline 291 & Alice & 69 & 51 & 249 & 56 & 4.316011 & 9 & 4 & 75 & & 46 & & & & \\
\hline 302 & Clarissa & 28 & -72 & 190 & -72 & 14.47670 & 8 & 2 & 102 & & 104 & & & & \\
\hline 310 & Margarita & 225 & -35 & 42 & -33 & 12.0710 & 27 & 1 & 88 & 31 & 51 & & & & \\
\hline 312 & Pierretta & 82 & -39 & 256 & -58 & 10.20764 & 4 & 1 & 176 & & 36 & 52 & & & \\
\hline 336 & Lacadiera & 194 & 39 & 37 & 54 & 13.69552 & 3 & 1 & 121 & & 36 & 32 & & & \\
\hline 340 & Eduarda & 188 & -43 & 18 & -47 & 8.00613 & 2 & 1 & 117 & & 76 & 31 & 36 & & \\
\hline 354 & Eleonora & 144 & 54 & & & 4.277186 & 37 & 9 & 258 & & 40 & & & 139 & 96 \\
\hline 355 & Gabriella & 341 & 78 & 197 & 70 & 4.82899 & 4 & 1 & 128 & & & & & & \\
\hline 367 & Amicitia & 203 & 38 & 21 & 32 & 5.05502 & 2 & 1 & 128 & & 34 & & & & \\
\hline 372 & Palma & 221 & -47 & 44 & 17 & 8.58189 & 28 & 6 & 214 & & 52 & 36 & & & \\
\hline 376 & Geometria & 239 & 45 & 63 & 53 & 7.71097 & 39 & 9 & 158 & & 76 & & & & \\
\hline 399 & Persephone & 36 & 63 & & & 9.14639 & & & 166 & & 36 & & & & \\
\hline 400 & Ducrosa & 328 & 56 & 158 & 62 & 6.86788 & 3 & 1 & 103 & & & & & & \\
\hline 413 & Edburga & 202 & -45 & & & 15.7715 & 2 & 1 & 148 & & 43 & & & & \\
\hline 436 & Patricia & 124 & -30 & 339 & -58 & 16.1320 & 4 & 1 & 97 & & 53 & 91 & & & \\
\hline 440 & Theodora & 80 & -88 & & & 4.83658 & & & 123 & & 103 & & 48 & & \\
\hline 471 & Papagena & 223 & 67 & 22 & 18 & 7.11539 & 13 & 2 & 293 & & 72 & & & 203 & 112 \\
\hline 486 & Cremona & 227 & 59 & 31 & 30 & 65.151 & 1 & 1 & 127 & & 55 & 35 & & & \\
\hline 499 & Venusia & 37 & 50 & 212 & 46 & 13.4871 & 4 & 1 & 122 & & 39 & & 31 & & \\
\hline 544 & Jetta & 275 & -84 & 31 & -67 & 7.74528 & 3 & 1 & 139 & & 60 & & & & \\
\hline 573 & Recha & 74 & -24 & 252 & -48 & 7.16586 & 3 & 1 & 161 & & 85 & & & & \\
\hline 584 & Semiramis & 106 & -56 & 315 & -32 & 5.06893 & 24 & 6 & 150 & & 59 & 49 & & & \\
\hline 590 & Tomyris & 273 & -47 & 120 & -46 & 5.55248 & 3 & 1 & 91 & & 32 & & & & \\
\hline 601 & Nerthus & 173 & 44 & 20 & 32 & 13.5899 & & & 139 & & 94 & & & & \\
\hline 606 & Brangane & 183 & 20 & 354 & 26 & 12.29067 & 2 & 1 & 108 & & 70 & & & & \\
\hline 629 & Bernardina & 40 & 33 & 236 & 48 & 3.76360 & & & 91 & & 48 & & & & \\
\hline 631 & Philippina & 183 & -2 & & & 5.90220 & 6 & 2 & 171 & & 38 & & & & \\
\hline 685 & Hermia & 197 & 87 & 29 & 79 & 50.387 & & & 93 & & 148 & & & & \\
\hline 695 & Bella & 87 & -55 & 314 & -56 & 14.21900 & 8 & 1 & 184 & & 90 & 30 & & & \\
\hline 753 & Tiflis & 5 & 36 & 199 & 57 & 9.8259 & & & 129 & & 64 & & & & \\
\hline 800 & Kressmannia & 345 & 37 & 172 & 34 & 4.460963 & 8 & 2 & 108 & & 51 & & & & \\
\hline 808 & Merxia & 26 & 54 & 192 & 57 & 30.630 & 4 & 1 & 158 & & 87 & & 32 & & \\
\hline 810 & Atossa & 12 & 67 & 188 & 69 & 4.38547 & & & 99 & & 71 & & 60 & & \\
\hline 825 & Tanina & 46 & 48 & 231 & 60 & 6.93981 & 2 & 1 & 114 & & 40 & & & & \\
\hline 832 & Karin & 242 & 46 & 59 & 44 & 18.3512 & 13 & 3 & 84 & & & 39 & & & \\
\hline 847 & Agnia & 341 & 18 & 162 & 13 & 14.8247 & 3 & 1 & 136 & & & & & & \\
\hline 889 & Erynia & 187 & -60 & 335 & -74 & 9.8749 & & & 94 & & 65 & & & & \\
\hline 925 & Alphonsina & 296 & 41 & 147 & 22 & 7.87754 & 4 & 1 & 134 & & 48 & 79 & & & \\
\hline 934 & Thuringia & 120 & -52 & & & 8.16534 & & & 123 & & 59 & & & & \\
\hline 1002 & Olbersia & 220 & 35 & 16 & 54 & 10.2367 & & & 87 & & 48 & 54 & & & \\
\hline 1087 & Arabis & 334 & -7 & 155 & 12 & 5.79501 & 3 & 1 & 156 & & 92 & & & & \\
\hline 1102 & Pepita & 25 & -34 & 231 & -30 & 5.10532 & & & 147 & & 47 & & & & \\
\hline 1140 & Crimea & 12 & -73 & 175 & -22 & 9.7869 & 3 & 1 & 96 & & 116 & & & & \\
\hline 1148 & Rarahu & 148 & -9 & 322 & -9 & 6.54449 & & & 95 & & 64 & & & & \\
\hline 1207 & Ostenia & 310 & -77 & 124 & -51 & 9.07129 & 2 & 2 & 87 & & 71 & & & & \\
\hline 1291 & Phryne & 106 & 35 & 277 & 59 & 5.58414 & 2 & 1 & 129 & & 72 & & & & \\
\hline 1301 & Yvonne & 39 & 41 & & & 7.31968 & & & 78 & & 56 & 33 & & & \\
\hline 1333 & Cevenola & 38 & -86 & 220 & -44 & 4.87932 & 3 & 1 & 104 & & 91 & & & & \\
\hline 1382 & Gerti & 268 & 23 & 87 & 28 & 3.081545 & 2 & 1 & 60 & & 56 & & 52 & & \\
\hline 1419 & Danzig & 22 & 76 & 193 & 62 & 8.11957 & 1 & 1 & 135 & & 87 & & & & \\
\hline
\end{tabular}


Table 3. continued.

\begin{tabular}{|c|c|c|c|c|c|c|c|c|c|c|c|c|c|c|c|}
\hline & $\overline{\text { Asteroid }}$ & $\begin{array}{c}\lambda_{1} \\
{[\mathrm{deg}]}\end{array}$ & $\begin{array}{c}\beta_{1} \\
{[\mathrm{deg}]}\end{array}$ & $\begin{array}{c}\lambda_{2} \\
{[\mathrm{deg}]}\end{array}$ & $\begin{array}{c}\beta_{2} \\
{[\mathrm{deg}]}\end{array}$ & $\begin{array}{c}P \\
{[\mathrm{~h}]}\end{array}$ & $\overline{\bar{N}}$ & 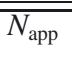 & $\bar{~} \overline{N_{689}}$ & $\bar{~} \overline{N_{699}}$ & 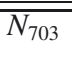 & $\overline{N_{\text {E12 }}}$ & $\bar{~} \overline{N_{\mathrm{G} 96}}$ & 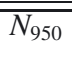 & 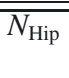 \\
\hline 1482 & Sebastiana & 262 & -68 & 91 & -67 & 10.48965 & 2 & 1 & 131 & & 39 & 30 & & & \\
\hline 1514 & Ricouxa & 251 & 75 & 68 & 69 & 10.42468 & 3 & 1 & 68 & & 56 & & & & \\
\hline 1568 & Aisleen & 109 & -68 & & & 6.67597 & & & 82 & & 37 & & & & \\
\hline 1635 & Bohrmann & 5 & -38 & 185 & -36 & 5.86427 & 8 & 1 & 108 & & 47 & & & & \\
\hline 1659 & Punkaharju & 259 & -71 & 75 & -22 & 5.01327 & 2 & 1 & 118 & & 66 & & & & \\
\hline 1682 & Karel & 232 & 32 & 51 & 41 & 3.37485 & & & 54 & & 84 & & 36 & & \\
\hline 1709 & Ukraina & 165 & -61 & 2 & -40 & 7.30517 & 2 & 1 & 46 & & 79 & & & & \\
\hline 1742 & Schaifers & 198 & 57 & 47 & 55 & 8.53270 & 3 & 1 & 106 & & & & & & \\
\hline 1747 & Wright & 227 & 31 & & & 5.28796 & & & 70 & & 55 & & & & \\
\hline 1889 & Pakhmutova & 22 & -76 & 167 & -40 & 17.5157 & & & 68 & & 46 & 35 & & & \\
\hline 1930 & Lucifer & 32 & 17 & 211 & -19 & 13.0536 & 6 & 1 & 106 & & 43 & 66 & & & \\
\hline 2156 & Kate & 49 & 74 & & & 5.62215 & 4 & 1 & & & 44 & & & & \\
\hline 3678 & Mongmanwai & 125 & -65 & & & 4.18297 & 2 & 1 & & & 103 & & 31 & & \\
\hline 4483 & Petofi & 107 & 40 & & & 4.33299 & 3 & 1 & & & 36 & & & & \\
\hline
\end{tabular}

Notes. For each asteroid, the table gives also the number of dense lightcurves $N_{\mathrm{lc}}$ observed during $N_{\text {app }}$ apparitions and the number of sparse data points for the corresponding observatory: $N_{689}, N_{699}, N_{703}, N_{\mathrm{E} 12}, N_{\mathrm{G} 96}, N_{950}$ and $N_{\mathrm{Hip}}$. Pole solutions preferred by asteroid occultation measurements (Ďrech et al. 2011) are emphasized by a bold font. 
J. Hanuš et al.: A study of asteroid pole-latitude distribution

Table 4. List of partial models derived from combined data sets.

\begin{tabular}{|c|c|c|c|c|c|c|c|c|c|c|c|c|c|}
\hline & Asteroid & $\begin{array}{c}\beta \\
{[\mathrm{deg}]}\end{array}$ & $\begin{array}{c}\Delta \\
{[\mathrm{deg}]}\end{array}$ & $\begin{array}{c}P \\
{[\mathrm{~h}]}\end{array}$ & $N_{\mathrm{lc}}$ & $\overline{N_{\text {app }}}$ & $\bar{N} N_{689}$ & $N_{699}$ & $N_{703}$ & $N_{\mathrm{E} 12}$ & $N_{\mathrm{G} 96}$ & $N_{950}$ & $N_{\text {Hip }}$ \\
\hline 163 & Erigone & -60 & 14 & 16.1403 & 3 & 1 & 168 & & 72 & & & & \\
\hline 187 & Lamberta & -58 & 9 & 10.66703 & 9 & 2 & 159 & & 52 & 53 & & & \\
\hline 233 & Asterope & 49 & 8 & 19.6981 & 13 & 3 & 184 & & 80 & & & 165 & \\
\hline 272 & Antonia & -70 & 6 & 3.85480 & 5 & 1 & 109 & & 60 & & 36 & & \\
\hline 281 & Lucretia & -54 & 11 & 4.349710 & 6 & 3 & 123 & 30 & 62 & & & & \\
\hline 313 & Chaldaea & 33 & 18 & 8.38992 & 9 & 3 & 176 & & 80 & & & & \\
\hline 390 & Alma & -60 & 22 & 3.74116 & 2 & 1 & 109 & & & 34 & & & \\
\hline 510 & Mabella & -59 & 12 & 19.4304 & 6 & 2 & 145 & & 60 & & & & \\
\hline 550 & Senta & -63 & 13 & 20.5726 & 9 & 1 & 151 & & & 61 & & & \\
\hline 622 & Esther & -61 & 9 & 47.5042 & 5 & 1 & 120 & & 60 & & & & \\
\hline 692 & Hippodamia & -52 & 25 & 8.99690 & 3 & 1 & 114 & & 78 & 32 & & & \\
\hline 733 & Mocia & 36 & 16 & 11.37611 & 2 & 1 & 175 & & 44 & & & & \\
\hline 746 & Marlu & -54 & 18 & 7.78887 & 3 & 1 & 133 & & 47 & 34 & & & \\
\hline 784 & Pickeringia & 58 & 15 & 13.1699 & 1 & 1 & 188 & & 67 & 32 & & & \\
\hline 823 & Sisigambis & 57 & 9 & 146.58 & 8 & 1 & 123 & & 90 & & & & \\
\hline 877 & Walkure & 53 & 12 & 17.4217 & 3 & 1 & 141 & 45 & 104 & & 32 & & \\
\hline 899 & Jokaste & -58 & 19 & 6.24812 & 3 & 1 & 140 & & & 43 & & & \\
\hline 1010 & Marlene & 46 & 7 & 31.066 & 8 & 1 & 104 & & 52 & & & & \\
\hline 1103 & Sequoia & -48 & 19 & 3.037977 & 2 & 1 & 111 & & 36 & 30 & & & \\
\hline 1185 & Nikko & 46 & 12 & 3.78615 & 3 & 1 & 91 & & 46 & 32 & & & \\
\hline 1188 & Gothlandia & -63 & 19 & 3.491820 & 2 & 1 & 129 & 33 & 67 & 41 & & & \\
\hline 1214 & Richilde & -59 & 15 & 9.86687 & 4 & 1 & 101 & & 78 & & & & \\
\hline 1282 & Utopia & -39 & 21 & 13.6228 & 4 & 1 & 116 & & 72 & & & & \\
\hline 1350 & Rosselia & -58 & 13 & 8.14011 & 1 & 1 & 114 & & 48 & & & & \\
\hline 1368 & Numidia & -50 & 14 & 3.640740 & 3 & 1 & 129 & & 47 & & & & \\
\hline 1379 & Lomonosowa & -62 & 17 & 24.4845 & 2 & 1 & 96 & & 100 & & & & \\
\hline 1389 & Onnie & -56 & 10 & 23.0447 & 2 & 1 & 85 & 33 & 47 & 32 & 40 & & \\
\hline 1665 & Gaby & 49 & 17 & 67.905 & 1 & 1 & 81 & & 80 & & & & \\
\hline 1719 & Jens & -56 & 19 & 5.87016 & 2 & 1 & 78 & & 48 & 40 & & & \\
\hline 2001 & Einstein & -51 & 22 & 5.4850 & 2 & 1 & & & 84 & & & & \\
\hline
\end{tabular}

Notes. For each asteroid, there is the mean ecliptic latitude $\beta$ of the pole direction and its dispersion $\Delta$, the other parameters have the same meaning as in Table 3.

Table 5. List of improved asteroid models that were originally designated in DAMIT as "preliminary".

\begin{tabular}{|c|c|c|c|c|c|c|c|c|c|c|c|c|c|c|c|}
\hline & Asteroid & $\begin{array}{c}\lambda_{1} \\
{[\mathrm{deg}]}\end{array}$ & $\begin{array}{c}\beta_{1} \\
{[\mathrm{deg}]}\end{array}$ & $\begin{array}{c}\lambda_{2} \\
{[\mathrm{deg}]}\end{array}$ & $\begin{array}{c}\beta_{2} \\
{[\mathrm{deg}]}\end{array}$ & $\begin{array}{c}P \\
{[\mathrm{~h}]}\end{array}$ & $\overline{N_{\mathrm{lc}}}$ & $N_{\text {app }}$ & $\overline{N_{689}}$ & $N_{699}$ & $N_{703}$ & $N_{\mathrm{E} 12}$ & $\overline{N_{\mathrm{G} 96}}$ & $N_{950}$ & $\overline{N_{\text {Hip }}}$ \\
\hline 73 & Klytia & 266 & 68 & 44 & 83 & 8.28307 & 21 & 7 & 131 & 36 & 98 & 47 & & & \\
\hline 82 & Alkmene & 164 & -28 & 349 & -33 & 13.00079 & 11 & 1 & 158 & & 72 & 36 & 38 & 192 & \\
\hline 132 & Aethra & 326 & 67 & & & 5.16827 & 4 & 2 & 204 & & 55 & & & & \\
\hline 152 & Atala & 347 & 46 & 199 & 61 & 6.24472 & 2 & 1 & 101 & & 32 & & & & \\
\hline 277 & Elvira & 121 & -84 & & & 29.6922 & 22 & 5 & 142 & & 36 & 51 & & & \\
\hline 278 & Paulina & 307 & 31 & 118 & 38 & 6.49387 & 3 & 1 & 195 & & 51 & & & & \\
\hline 311 & Claudia & 214 & 43 & 30 & 40 & 7.53138 & 23 & 6 & 114 & 33 & 108 & & 40 & & \\
\hline 484 & Pittsburghia & 70 & 46 & & & 10.64977 & 2 & 1 & 100 & & 52 & & & & \\
\hline 516 & Amherstia & 254 & 22 & 81 & 54 & 7.48431 & 5 & 3 & 162 & & 32 & & & & \\
\hline 534 & Nassovia & 66 & 41 & 252 & 42 & 9.46889 & 16 & 6 & 151 & & 64 & 32 & & & \\
\hline 614 & Pia & 348 & 48 & 162 & 27 & 4.57872 & 2 & 1 & 121 & & 78 & & & & \\
\hline 714 & Ulula & 224 & -10 & 41 & -5 & 6.99837 & 9 & 2 & 177 & & 67 & & & & \\
\hline 770 & Bali & 70 & 50 & 262 & 45 & 5.81894 & 2 & 1 & 131 & & 52 & & & & \\
\hline 915 & Cosette & 350 & 56 & 189 & 61 & 4.469742 & 1 & 1 & 106 & & 32 & 35 & & & \\
\hline 1012 & Sarema & 45 & 67 & 253 & 63 & 10.30708 & 2 & 1 & 74 & & 42 & & & & \\
\hline 1022 & Olympiada & 46 & 10 & 242 & 52 & 3.83359 & 5 & 2 & 107 & & 91 & & & & \\
\hline 1088 & Mitaka & 280 & -71 & & & 3.035378 & 1 & 1 & 104 & & 39 & 41 & & & \\
\hline 1223 & Neckar & 252 & 28 & 69 & 30 & 7.82401 & 16 & 7 & 132 & 33 & 60 & & 36 & & \\
\hline
\end{tabular}

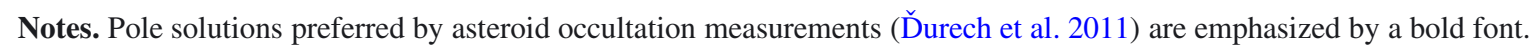


A\&A 530, A134 (2011)

Table 6. Observations used for successful model determination that are not included in the UAPC.

\begin{tabular}{|c|c|c|c|c|c|c|c|}
\hline$\overline{\text { Aste }}$ & eroid & $\overline{\text { Date }}$ & Observer & & Asteroid & $\overline{\text { Date }}$ & Observer \\
\hline 13 & Egeria & $2007.9-2009.3$ & Pilcher (2009) & 808 & Merxia & 2003126.8 & Casulli $^{a}$ \\
\hline \multirow[t]{2}{*}{14} & Irene & $2007.11-2009.5$ & Pilcher (2009) & & & 2003128.8 & Casulli $^{a}$ \\
\hline & & 200823.7 & Polishook & & & 200327.0 & Bernasconi $^{a}$ \\
\hline \multirow[t]{2}{*}{40} & Harmonia & 2008.12-2009.1 & Pilcher (2009) & & & 200328.0 & Bernasconi $^{a}$ \\
\hline & & $2010.5-2010.6$ & Pilcher (2010) & 832 & Karin & $2003.8-2003.9$ & Yoshida et al. (2004) \\
\hline \multirow[t]{3}{*}{68} & Leto & 2008126.0 & Pilcher ${ }^{b}$ & & & $2004.9-2004.9$ & Ito \& Yoshida (2007) \\
\hline & & 2008130.0 & Pilcher $^{b}$ & 899 & Jokaste & $2003.11-2003.12$ & Stephens (2004) \\
\hline & & 200826.1 & Pilcher $^{b}$ & 1010 & Marlene & $2005.1-2005.3$ & Warner (2005a) \\
\hline \multirow[t]{2}{*}{264} & Libussa & $2005.2-2005.3$ & Pilcher \& Cooney (2006) & 1022 & Olympiada & $1999.6-1999.6$ & Warner (2005b) \\
\hline & & $2008.10-2008.12$ & Pilcher \& Jardine (2009) & 1087 & Arabis & 2003223.0 & Lehký \\
\hline 272 & Antonia & $2007.12-2008.1$ & Pilcher (2008) & 1140 & Crimea & $2005.4-2005.4$ & Stephens (2005a) \\
\hline 310 & Margarita & $2010.3-2010.5$ & Pilcher \& Oey (2010) & 1185 & Nikko & $2004.11-2004.11$ & Stephens (2005b) \\
\hline 390 & Alma & $2004.8-2004.8$ & Stephens $(2005 \mathrm{c})$ & 1282 & Utopia & $2000.11-2000.11$ & Warner (2011b) \\
\hline 400 & Ducrosa & $2005.1-2005.1$ & Warner (2005a) & 1333 & Cevenola & $2002.2-2002.2$ & Warner (2002) \\
\hline 436 & Patricia & $2002.12-2003.1$ & Warner (2003) & 1635 & Bohrmann & $2003.9-2003.10$ & Stephens \& Warner (2004) \\
\hline 544 & Jetta & $2004.8-2004.8$ & Stephens $(2005 c)$ & 1659 & Punkaharju & $2000.11-2000.11$ & Warner (2011c) \\
\hline 573 & Recha & $2001.1-2001.1$ & Warner (2011b) & 1719 & Jens & $2000.9-2000.9$ & Warner (2011c) \\
\hline \multirow[t]{5}{*}{714} & Ulula & 2005923.8 & Henych & 1930 & Lucifer & $2003.10-2003.10$ & Warner (2005a) \\
\hline & & 2005925.8 & Henych & 2001 & Einstein & $2004.12-2004.12$ & Warner (2005a) \\
\hline & & 2005930.8 & Henych & 3678 & Mongmanwai & $2003.3-2003.3$ & Stephens (2003) \\
\hline & & 2005101.8 & Henych & & & & \\
\hline & & 20051010.8 & Henych & & & & \\
\hline
\end{tabular}

Notes. ${ }^{(a)}$ Online at http://obswww.unige.ch/ behrend/page_cou.html. ${ }^{(b)}$ Online at http://aslc-nm.org/Pilcher.html 